Annales Academiæ Scientiarum Fennicæ

Mathematica

Volumen 38, 2013, 631-656

\title{
LYAPUNOV SPECTRUM FOR EXCEPTIONAL RATIONAL MAPS
}

\author{
Katrin Gelfert, Feliks Przytycki, Michał Rams and Juan Rivera-Letelier
}

\author{
Universidade Federal do Rio de Janeiro, Instituto de Matemática \\ Cidade Universitária - Ilha do Fundão, Rio de Janeiro 21945-909, Brazil; gelfert@im.ufrj.br \\ Instytut Matematyczny Polskiej Akademii Nauk \\ ul. Śniadeckich 8, 00-956 Warszawa, Poland; feliksp@impan.gov.pl \\ Instytut Matematyczny Polskiej Akademii Nauk \\ ul. Śniadeckich 8, 00-956 Warszawa, Poland; rams@impan.gov.pl \\ Pontificia Universidad Católica de Chile, Facultad de Matemáticas \\ Avenida Vicuña Mackenna 4860, Santiago, Chile; riveraletelier@mat.puc.cl
}

\begin{abstract}
We study the dimension spectrum for Lyapunov exponents for rational maps acting on the Riemann sphere and characterize it by means of the Legendre-Fenchel transform of the hidden variational pressure. This pressure is defined by means of the variational principle with respect to nonatomic invariant probability measures and is associated to certain $\sigma$-finite conformal measures. This allows to extend previous results to exceptional rational maps.
\end{abstract}

\section{Introduction and main results}

We are going to study the Lyapunov exponents of a rational function $f: \overline{\mathbf{C}} \rightarrow \overline{\mathbf{C}}$ acting on the Riemann sphere, of degree at least 2. In particular, continuing the investigations in [7], we are interested in the case that the map $f$ is exceptional. Slightly modifying [10, Section 1.3], we call $f$ exceptional if there exists a finite, nonempty, and forward invariant set $\Sigma^{\prime} \subset J$ such that

$$
f^{-1}\left(\Sigma^{\prime}\right) \backslash \Sigma^{\prime} \subset \text { Crit . }
$$

Here $J=J(f)$ is the Julia set of $f$ and Crit $=\operatorname{Crit}(f)$ is the set of critical points of $f$. Every such set $\Sigma^{\prime}$ has at most 4 points (see Lemma 1), hence there is a maximal set with this property, which we denote by $\Sigma(f)$. If $f$ is non-exceptional we put $\Sigma(f)=\varnothing$. When $f$ is clear from the context we denote $\Sigma(f)$ simply by $\Sigma$.

1.1. Main results. Given $x \in J$, denote by $\chi(x)$ and $\bar{\chi}(x)$ the lower and upper Lyapunov exponent at $x$, respectively. If both values coincide then we call the common value the Lyapunov exponent at $x$ and denote it by $\chi(x)$. Similarly, for a $f$-invariant probability measure $\mu$ we denote by $\chi(\mu) \stackrel{\text { def }}{=} \int \log \left|f^{\prime}\right| d \mu$ its Lyapunov exponent. Let $\mathcal{M}$ be the set of all $f$-invariant Borel probability measures supported on $J$ and $\widetilde{\mathcal{M}} \subset \mathcal{M}$ be the one of all nonatomic ones. Let $\widetilde{\mathcal{M}}_{E}$ and $\mathcal{M}_{E}$ be the sets of ergodic measures contained in $\widetilde{\mathcal{M}}$ and $\mathcal{M}$, respectively. Let

$$
\alpha^{-} \stackrel{\text { def }}{=} \inf _{\mu \in \mathcal{M}_{E}} \chi(\mu), \quad \alpha^{+} \stackrel{\text { def }}{=} \sup _{\mu \in \mathcal{M}_{E}} \chi(\mu) \quad \text { and } \quad \widetilde{\alpha}^{+} \stackrel{\text { def }}{=} \sup _{\mu \in \widetilde{\mathcal{M}}_{E}} \chi(\mu)
$$

doi:10.5186/aasfm.2013.3849

2010 Mathematics Subject Classification: Primary 37D25, 37D35, 37C45, 28D99, 37F10.

Key words: Lyapunov exponents, exceptional rational maps, pressure, conformal measures, Lyapunov spectrum, multifractal formalism. 
(see Corollary 1 for equivalent definitions of $\widetilde{\alpha}^{+}$).

For given numbers $0 \leq \alpha \leq \beta$, we consider the level sets

$$
\mathcal{L}(\alpha, \beta) \stackrel{\text { def }}{=}\{x \in J: \underline{\chi}(x)=\alpha, \bar{\chi}(x)=\beta\} .
$$

If $\alpha<\beta$ then $\mathcal{L}(\alpha, \beta)$ is contained in the set of so-called irregular points. We denote by $\mathcal{L}(\alpha) \stackrel{\text { def }}{=} \mathcal{L}(\alpha, \alpha)$ the set of Lyapunov regular points with exponent $\alpha$. We will describe the complexity of such level sets in terms of their Hausdorff dimension $\operatorname{dim}_{\mathrm{H}}$. To do so, given a parameter $t \in \mathbf{R}$ let us consider the potential $\varphi_{t} \stackrel{\text { def }}{=}-t \log \left|f^{\prime}\right|$ and the pressure function

$$
P\left(\varphi_{t}\right) \stackrel{\text { def }}{=} \sup _{\mu \in \mathcal{M}}\left(h_{\mu}(f)+\int_{J} \varphi_{t} d \mu\right),
$$

see [15] for further details on the definition of pressure. We define the hidden variational pressure

$$
\widetilde{P}\left(\varphi_{t}\right) \stackrel{\text { def }}{=} \sup _{\mu \in \widetilde{\mathcal{M}}}\left(h_{\mu}(f)+\int_{J} \varphi_{t} d \mu\right)
$$

(following the terminology in [10]). After Makarov and Smirnov [10, Theorem B], the pressure function $t \mapsto P\left(\varphi_{t}\right)$ fails to be real analytic on the interval $(-\infty, 0)$ if and only if $f$ is exceptional and $\alpha^{+}>\widetilde{\alpha}^{+}$. By [10, Theorem A], for an arbitrary rational function the hidden pressure function $t \mapsto \widetilde{P}\left(\varphi_{t}\right)$ is real analytic on the interval $(-\infty, 0)$ and

$$
P\left(\varphi_{t}\right)=\max \left\{\widetilde{P}\left(\varphi_{t}\right),-t \alpha^{+}\right\}
$$

For any $\alpha>0$ let

$$
\widetilde{F}(\alpha) \stackrel{\text { def }}{=} \frac{1}{\alpha} \inf _{t \in \mathbf{R}}\left(\widetilde{P}\left(\varphi_{t}\right)+t \alpha\right) \quad \text { and } \quad \widetilde{F}(0) \stackrel{\text { def }}{=} \lim _{\alpha \rightarrow 0+} \widetilde{F}(\alpha) .
$$

This function is finite and non-negative on $\left[\alpha^{-}, \widetilde{\alpha}^{+}\right]$, and equal to $-\infty$ on $\mathbf{R} \backslash\left[\alpha^{-}, \widetilde{\alpha}^{+}\right]$.

Our main result is the following theorem.

Theorem 1. Let $f$ be a rational function of degree at least 2. For any $\alpha$ in $\left[\alpha^{-}, \widetilde{\alpha}^{+}\right] \backslash\{0\}$, we have

$$
\operatorname{dim}_{\mathrm{H}} \mathcal{L}(\alpha)=\widetilde{F}(\alpha),
$$

and for every $\beta$ in $\left[\alpha, \widetilde{\alpha}^{+}\right]$, we have

$$
\min \{\widetilde{F}(\alpha), \widetilde{F}(\beta)\} \leq \operatorname{dim}_{\mathrm{H}} \mathcal{L}(\alpha, \beta) \leq \max _{\alpha \leq q \leq \beta} \widetilde{F}(q) .
$$

For $\alpha=0$ we also have

$$
\operatorname{dim}_{\mathrm{H}} \mathcal{L}(0) \geq \widetilde{F}(0)
$$

Moreover,

$$
\left\{x \in J:-\infty<\chi(x)<\alpha^{-}\right\}=\left\{x \in J \backslash \Sigma: \bar{\chi}(x)>\widetilde{\alpha}^{+}\right\}=\varnothing
$$

and in the case $\alpha^{-}>0$ we also have

$$
\operatorname{dim}_{\mathrm{H}}\left\{x \in J: \underline{\chi}(x)>0, \bar{\chi}(x)<\alpha^{-}\right\}=0 .
$$


The result of the above theorem has been shown in [7] in the particular case that $f$ is non-exceptional.

The multifractal formalism for Lyapunov exponents for conformal uniformly expanding repellers has been covered for the first time by Barreira, Pesin, and Schmeling [1] building also on work by Weiss [18] (see the monographs [12, 16] for more details and references). To our best knowledge, the first results on irregular parts of a spectrum were obtained by Besicovitch [3]. Its first complete description (for digit expansions) was given in [2].

To prove our main result, in this paper we will create new technical tools in order to deal with exceptional rational maps and then show how these tools can be applied to adapt the original proofs in [7]. The paper is organized as follows. In Section 2 we collect some known results about exceptional maps that will be used in the rest of the paper. In Section 3 we will introduce the concept of hidden pressure using backward branches of $f$, analogously to the tree pressure from [15]. In the case of exceptional rational maps we do not always have at hand a finite conformal measure with dense support, see Proposition 1. For that reason, in Section 4 we introduce $\sigma$-finite conformal measures that are associated to the hidden pressure. Finally, in Section 5 we apply these tools to prove Theorem 1. In Section 5.1 we provide a lower bound for dimension using the fact that for any rational map we can find an increasing family of uniformly expanding Cantor repellers contained in $J$ using a construction of bridges that has been established in [7] and applies to the setting of this paper without changes. In Section 5.2 we provide an upper bound for dimension applying Frostman's Lemma to an appropriate $\sigma$-finite conformal measure at a conical point. Finally, in Section 5.3, we show the existence of periodic orbits in $J \backslash \Sigma$ with exponent as large as possible. The proof of Theorem 1 is given at the end of Section 5.3.

We give an alternative proof of this result in Appendix A via a variant of Bowen's periodic specification property, [4].

Throughout the rest of this paper we fix a rational map $f$ of degree at least 2 , and denote the spherical distance on the Riemann sphere $\overline{\mathbf{C}}$ by dist. Given an integer $a \geq 1$ and a function $g$ defined on $J$, put

$$
S_{a} g \stackrel{\text { def }}{=} g+g \circ f+\cdots+g \circ f^{a-1} .
$$

\section{Exceptional maps and phase transitions}

For a critical point $c \in$ Crit we will denote by $\operatorname{deg}_{f}(c)$ the local degree of $f$ at $z=c$. The following result has been proved by the same computation first in $[6$, Lemma 2].

Lemma 1. If $\Sigma^{\prime}$ is a finite subset of $\overline{\mathbf{C}}$ such that $f^{-1}\left(\Sigma^{\prime}\right) \backslash \Sigma^{\prime} \subset$ Crit, then $\operatorname{card} \Sigma^{\prime} \leq 4$. If $f$ is a polynomial then $\operatorname{card}\left(\Sigma^{\prime} \backslash\{\infty\}\right) \leq 2$.

Proof. Using that $f$ has $2 \operatorname{deg}(f)-2$ critical points counted with multiplicity, by (1) we have

$$
\begin{aligned}
\operatorname{deg}(f) \operatorname{card} \Sigma^{\prime} & =\sum_{x \in f^{-1}\left(\Sigma^{\prime}\right)} \operatorname{deg}_{f}(x)=\operatorname{card} f^{-1}\left(\Sigma^{\prime}\right)+\sum_{x \in f^{-1}\left(\Sigma^{\prime}\right)}\left(\operatorname{deg}_{f}(x)-1\right) \\
& \leq \operatorname{card} \Sigma^{\prime}+\operatorname{card} \text { Crit }+2(\operatorname{deg}(f)-1) \leq \operatorname{card} \Sigma^{\prime}+4(\operatorname{deg}(f)-1),
\end{aligned}
$$


so card $\Sigma^{\prime} \leq 4$. If $f$ is a polynomial, then it has at most $\operatorname{deg}(f)-1$ finite critical points counted with multiplicity, so in this case $\operatorname{card}\left(\Sigma^{\prime} \backslash\{\infty\}\right) \leq 2$.

The following is an example of a one parameter family of rational maps, such that for some parameters the map is exceptional and its exceptional set contains a critical point. See [9, Section 6] and [10, Section 1.3] for other examples of exceptional rational maps.

Example 1. Let $d \geq 3$ be an integer, and for $\lambda \in \mathbf{C}$ put

$$
f_{\lambda}(z) \stackrel{\text { def }}{=}\left(\lambda z^{d}-\lambda z^{d-1}+1\right)^{-1} \text {. }
$$

The point $z=0$ is critical of multiplicity $d-2$, the point $1=f_{\lambda}(0)$ is fixed of multiplier $-\lambda$, and the point $z=\infty$ is critical of multiplicity $d-1$ and the only preimage of $z=0$. Thus, when $z=1$ belongs to the Julia set we have $\{0,1\} \subset \Sigma$. There are three cases when this last property can happen: When $|\lambda|>1(z=1$ is repelling), when $\lambda$ is a root of unity ( $z=1$ is parabolic), and the remaining case when $|\lambda|=1, \lambda$ is not a root of unity, and $f_{\lambda}$ is not locally linearizable at $z=1$ $(z=1$ is Cremer).

If $f$ is exceptional, then the set $\Sigma$ contains at least one periodic point. Observe that it hence must consist of a finite number of periodic points plus possibly some of their preimages. We write $\Sigma=\Sigma_{0} \cup \Sigma_{+}$, where $\Sigma_{0}$ denotes the subset of all neutral periodic points in $\Sigma$ plus its preimages and where $\Sigma_{+}$denotes the subset of all repelling periodic points in $\Sigma$ plus its preimages.

We will say that $f$ has a phase transition in the negative spectrum if the function $t \mapsto P\left(\varphi_{t}\right)$ fails to be real analytic on $(-\infty, 0)$. In this case we put

$$
t_{-} \stackrel{\text { def }}{=} \sup \left\{t<0: P\left(\varphi_{t}\right)=-t \alpha^{+}\right\} \text {. }
$$

We have $t_{-}<0$ and, since the function $t \mapsto P\left(\varphi_{t}\right)$ is convex, for each $t \in\left(-\infty, t_{-}\right)$ we have $P\left(\varphi_{t}\right)=-t \alpha^{+}$.

In the following proposition we gather several results in [10, 15]. A measurable subset $A$ of $\overline{\mathbf{C}}$ is said to be special if $f: A \rightarrow f(A)$ is injective. Given a measurable function $\psi: \overline{\mathbf{C}} \rightarrow[-\infty,+\infty]$, a (possibly infinite) Borel measure $\nu$ on $J$ is said to be $e^{\psi}$-conformal outside $Z \subset J$ if for every special set $A \subset J \backslash Z$ we have

$$
\nu(f(A))=\int_{A} e^{\psi(x)} d \nu(x) .
$$

If $Z=\varnothing$ we simply say that $\nu$ is $e^{\psi}$-conformal.

Proposition 1. Let $f$ be a rational map of degree at least 2 and let $t \in \mathbf{R}$. Then we have the following properties:

1. Suppose that $f$ does not have a phase transition in the negative spectrum, or that $f$ has a phase transition in the negative spectrum and $t>t_{-}$. Then $\widetilde{P}\left(\varphi_{t}\right)=$ $P\left(\varphi_{t}\right)$ and there is a finite $\left(e^{P\left(\varphi_{t}\right)-\varphi_{t}}\right)$-conformal measure whose support is equal to $J$;

2. Suppose that $f$ has a phase transition in the negative spectrum and that $t \leq t_{-}$. Then $f$ is exceptional, there is a repelling periodic point $p \in \Sigma$ such that $P\left(\varphi_{t}\right)=-t \chi(p)$ and for every neighborhood $V$ of $p$ and every measure $\nu$ that is $\left(e^{P\left(\varphi_{t}\right)-\varphi_{t}}\right)$-conformal outside Crit and that is not supported on $\Sigma$, we have

$$
\nu(V \backslash\{p\})=+\infty .
$$


Proof. The equality $\widetilde{P}\left(\varphi_{t}\right)=P\left(\varphi_{t}\right)$ in part 1 follows from the definition of $t_{-}$. The existence of the conformal measure in part 1 follows from [10, Lemma 3.5] if $t<0$ and from [15, Theorem A] if $t \geq 0$.

The fact that $f$ is exceptional and that there is a repelling periodic point $p \in \Sigma$ such that $P\left(\varphi_{t}\right)=-t \chi(p)$ in part 2 is given by [10, Theorem B]. To complete the proof of part 2 , let $\nu$ be a $\left(e^{P\left(\varphi_{t}\right)-\varphi_{t}}\right)$-conformal measure outside Crit that is not supported on $\Sigma$. We first prove that the support of $\nu$ is equal to $J$. Since by hypothesis $\nu$ is not supported on $\Sigma$, there is a point $z_{0}$ of $J \backslash \Sigma$ in the support of $\nu$. It follows that there is a sequence of points $\left(z_{n}\right)_{n=1}^{\infty}$ in $J \backslash$ Crit not contained in a periodic orbit, and such that for every $n$ we have $f\left(z_{n}\right)=z_{n-1}$. The conformality of $\nu$ outside Crit implies that for every $n \geq 0$ the point $z_{n}$ is in the support of $\nu$. It follows that there is an integer $N \geq 0$ such that $z_{N}$ is not in the forward orbit of a critical point of $f$. Using the conformality of $\nu$ outside Crit again, we conclude that every iterated preimage of $z_{N}$ is in the support of $\nu$. Since the iterated preimages of $z_{N}$ are dense in $J$, it follows that the support of $\nu$ is equal to $J$, as claimed.

Let $n \geq 1$ be the period of the periodic point $p$ and let $r>0$ be sufficiently small so that $B(p, r) \backslash\{p\} \subset V \backslash(\Sigma \cup$ Crit $)$, and so that the inverse branch $\phi$ of $f^{n}$ fixing $p$ is defined on a neighborhood of $\overline{B(p, r)}$ and satisfies $\phi(\overline{B(p, r)}) \subset B(p, r)$. Note that if we put $U \stackrel{\text { def }}{=} B(p, r) \backslash \phi(B(p, r))$, then we have $\nu(U)>0$; otherwise the conformality of $\nu$ outside Crit would imply that for every integer $m \geq 1$ we have $\nu\left(\phi^{m}(U)\right)=0$, and therefore

$$
\nu(B(p, r) \backslash\{p\})=\sum_{m=0}^{\infty} \nu\left(\phi^{m}(U)\right)=0,
$$

but this is not possible because the open set $B(p, r) \backslash\{p\}$ intersects $J$ and the support of $\nu$ is equal to $J$. Since $\phi$ is defined on a neighborhood of $\overline{B(p, r)}$ and $\phi(\overline{B(p, r)}) \subset$ $B(p, r)$, there is a distortion constant $C>0$ such that for each integer $m \geq 1$ we have, by the conformality of $\nu$ outside Crit,

$$
\nu\left(\phi^{m}(U)\right) \geq C^{-1} \nu(U) e^{-m\left(n P\left(\varphi_{t}\right)-S_{n} \varphi_{t}(p)\right)}=C^{-1} \nu(U) .
$$

Thus

$$
\nu(V \backslash\{p\}) \geq \nu(B(p, r) \backslash\{p\})=\sum_{m=0}^{\infty} \nu\left(\phi^{m}(U)\right)=+\infty,
$$

completing the proof of the proposition.

\section{Hidden tree pressure}

The goal of this section is to prove equivalence of three pressure functions: The hidden variational pressure defined in (3) as well as the hidden hyperbolic pressure and the hidden tree pressure defined in (8) and (10) below.

Given $t \in \mathbf{R}$, the hidden hyperbolic pressure is defined as

$$
\widetilde{P}_{\text {hyp }}\left(\varphi_{t}\right) \stackrel{\text { def }}{=} \sup P_{f \mid X}\left(\varphi_{t}\right)
$$

where the supremum is taken over all compact $f$-invariant (i.e. $f(X) \subset X$ ) isolated expanding subsets of $J \backslash \Sigma$. We call such a set uniformly expanding repeller. Here isolated means that there exists a neighborhood $U$ of $X$ such that $f^{n}(x) \in U$ for all $n \geq 0$ implies $x \in X$.

Proposition 2. $\widetilde{P}\left(\varphi_{t}\right)=\widetilde{P}_{\text {hyp }}\left(\varphi_{t}\right)$ for every $t \in \mathbf{R}$. 
Proof. The inequality $\widetilde{P}\left(\varphi_{t}\right) \geq \widetilde{P}_{\text {hyp }}\left(\varphi_{t}\right)$ follows from the variational principle. On the other hand [16, Theorem 11.6.1] implies that for any $\mu \in \widetilde{\mathcal{M}}$ we have $\widetilde{P}_{\text {hyp }}\left(\varphi_{t}\right) \geq$ $h_{\mu}(f)+\int_{J} \varphi_{t} d \mu$ and hence $\widetilde{P}_{\text {hyp }}\left(\varphi_{t}\right) \geq \widetilde{P}\left(\varphi_{t}\right)$.

Before defining the hidden tree pressure, let us recall some concepts from [14], [15], and [16, Chapter 12.5]. Given $z \in \overline{\mathbf{C}}$ and $t \in \mathbf{R}$, we consider the tree pressure of $\varphi_{t}$ at $z$ defined by

$$
P_{\text {tree }}\left(z, \varphi_{t}\right) \stackrel{\text { def }}{=} \limsup _{n \rightarrow \infty} \frac{1}{n} \log \sum_{x \in f^{-n}(z)}\left|\left(f^{n}\right)^{\prime}(x)\right|^{-t} .
$$

A point $z \in \overline{\mathbf{C}}$ is said to be safe if

$$
z \notin \bigcup_{n=1}^{\infty} f^{n}(\text { Crit }) \quad \text { and } \quad \lim _{n \rightarrow \infty} \frac{1}{n} \log \operatorname{dist}\left(z, f^{n}(\text { Crit })\right)=0 .
$$

A point $z \in \overline{\mathbf{C}}$ is said to be expanding if there exist numbers $\Delta>0$ and $\lambda>1$ such that for all sufficiently large $n$ the map $f^{n}$ is univalent on $f_{z}^{-n}\left(B\left(f^{n}(z), \Delta\right)\right)$ and satisfies $\left|\left(f^{n}\right)^{\prime}(z)\right| \geq \lambda^{n}$. Here, for a subset $U$ of $\overline{\mathbf{C}}$ and $z \in U$ we denote by $f_{z}^{-n}(U)$ the connected component of $f^{-n}(U)$ containing $z$.

We point out that every point in $\overline{\mathbf{C}}$ outside a set of Hausdorff dimension zero is safe, and that for each safe point $z \in \overline{\mathbf{C}}$ we have $P_{\text {tree }}\left(z, \varphi_{t}\right)=P\left(\varphi_{t}\right)$, see $[15$, Theorem A], and compare with [14, Theorem 3.4]. Moreover, there is at least one safe point in $J \backslash \Sigma$ that is also expanding, see for example [16, Proposition 12.5.10].

Let us now define the hidden tree pressure that is an analogue of the tree pressure, obtained by considering a restricted tree of preimages. Given a subset $V$ of $J$ and $z \in$ $J \backslash V$ which is not in the forward orbit of a critical point, we define

$$
P_{n}\left(z, \varphi_{t}, V\right) \stackrel{\text { def }}{=} \frac{1}{n} \log \sum_{x \in f^{-n}(z) \cap J \backslash V}\left|\left(f^{n}\right)^{\prime}(x)\right|^{-t}
$$

and we consider the hidden tree pressure of $\varphi_{t}$ at $z$ defined by

$$
P_{\text {tree }}\left(z, \varphi_{t}, V\right) \stackrel{\text { def }}{=} \limsup _{n \rightarrow \infty} P_{n}\left(z, \varphi_{t}, V\right)
$$

Usually the point $z$ will be expanding safe in $J \backslash \Sigma$, and $V$ a neighborhood of $\Sigma$ not containing $z$.

Lemma 2. If $t \leq 0, V$ is a sufficiently small neighborhood of $\Sigma$, and $z \in J \backslash V$ is expanding safe, then the pressure $P_{\text {tree }}\left(z, \varphi_{t}, V\right)$ does not depend on $V$.

To prove the above lemma we need the following technical lemma.

Lemma 3. For an arbitrary neighborhood $V$ of $\Sigma$ and an arbitrary number $\varepsilon>0$ there exists a number $\delta>0$ and positive integers $N \leq M$ such that for every point $x \in J \backslash V$ there exist numbers $0 \leq i, j \leq M$ and a point $z \in f^{-j}\left(f^{i}(\{x\})\right)$ such that the set $A \stackrel{\text { def }}{=} f^{-N}(\{z\})$ is $\varepsilon$-dense in $J$ and satisfies

$$
\operatorname{dist}\left(\bigcup_{s=0}^{N+j-1} f^{s}(A), \text { Crit }\right) \geq \delta
$$


Proof. By the locally eventually onto property of $f$ on $J$ there is an integer $N \geq 1$ such that for each $z \in J$ the set $f^{-N}(z)$ is $\varepsilon$-dense in $J$. We put

$$
C(N) \stackrel{\text { def }}{=} \bigcup_{s=1}^{N} f^{s} \text { (Crit). }
$$

For each integer $M \geq 0$ let $\Phi_{M}: J \rightarrow \mathbf{R}$ be defined by

$$
\begin{gathered}
\Phi_{M}(x) \stackrel{\text { def }}{=} \max \left\{\min \left\{\operatorname{dist}(y, C(N)), \operatorname{dist}\left(\bigcup_{s=0}^{j-1} f^{s}(y), \text { Crit }\right)\right\}:\right. \\
\left.0 \leq i, j \leq M, y \in f^{-j}\left(f^{i}(x)\right)\right\} .
\end{gathered}
$$

We will show that for each $x \in J \backslash \Sigma$ there is an integer $M(x) \geq 0$ such $\Phi_{M(x)}(x)>0$. Since for each $M \geq 0$ the function $\Phi_{M}$ is continuous and for each $x \in J$ the sequence $\left(\Phi_{M}(x)\right)_{M=0}^{\infty}$ is nondecreasing, it follows that there is a number $M \geq N$ so that $\Phi_{M}$ is strictly positive on $J \backslash \Sigma$. This will imply the desired assertion with

$$
\delta \stackrel{\text { def }}{=} \inf \left\{\Phi_{M}(x): x \in J \backslash V\right\} \cdot\left(\sup \left|f^{\prime}(x)\right|\right)^{-N} .
$$

We distinguish three cases:

1) If $x \in J \backslash \Sigma$ is not in the forward orbit of a critical point then $\Phi_{0}(x)>0$.

2) If $x \in J \backslash \Sigma$ is in the forward orbit of a critical point that is not preperiodic then there exists a number $i=i(x) \in\{0, \ldots$, card $C(N)\}$ such that $f^{i}(x)$ is disjoint from $C(N)$. Hence, we obtain that $\Phi_{\text {card } C(N)}(x)>0$.

3) If $x \in J \backslash \Sigma$ is in the forward orbit of a preperiodic critical point then, there is $i$ and an infinite backward trajectory starting at $f^{i}(x)$ that is disjoint from Crit and in particular this backward trajectory is longer than card $C(N)$. Hence, we can choose numbers $i=i(x), j=j(x) \geq 0$ and a point $y=y(x) \in f^{-j}\left(f^{i}(x)\right)$ such that $y$ is not in the forward orbit of a critical point and such that for each $s \in\{0, \ldots, j-1\}$ we have $f^{s}(y) \notin$ Crit. In particular, we have $y \notin C(N)$. Thus, if we put

$$
M(x) \stackrel{\text { def }}{=} \max \{i(x), j(x)\},
$$

then $\Phi_{M(x)}(x)>0$.

Proof of Lemma 2. Let $V_{1}, V_{2}$ be two neighborhoods of $\Sigma$. Without loss of generality we can assume that $V_{1} \subset V_{2}$. By Lemma 3, every backward branch of $f^{-n}$ starting at $z$ and ending at some point $x_{1} \in V_{2} \backslash V_{1}$ can be modified to end at some $x_{2} \notin V_{2}$. The modification involves only removing at most $M$ last steps, that decreases $\left|\left(f^{n}\right)^{\prime}(x)\right|^{-t}$ at most by a constant factor because $t \leq 0$, and replacing them by at most $M+N$ steps, which stay in a uniformly bounded from below distance from critical points. Hence we conclude that $P_{n}\left(z, \varphi_{t}, V_{1}\right)$ and $P_{n}\left(z, \varphi_{t}, V_{2}\right)$ differ at most by $\mathcal{O}\left(n^{-1}\right)$. This proves the lemma.

We denote by Dist $\left.g\right|_{Z} \stackrel{\text { def }}{=} \sup _{x, y \in Z}\left|g^{\prime}(x)\right| /\left|g^{\prime}(y)\right|$ the maximal distortion of a map $g$ on a set $Z$. We establish one preliminary approximation result.

Proposition 3. Given $t \leq 0$, a sufficiently small neighborhood $V$ of $\Sigma$ and an expanding safe point $z \in J \backslash \Sigma$, for every $\varepsilon>0$ there exists a uniformly expanding repeller $X \subset J \backslash \Sigma$ such that

$$
P_{f \mid X}\left(\varphi_{t}\right) \geq P_{\text {tree }}\left(z, \varphi_{t}, V\right)-\varepsilon
$$


Proof. We start by recalling the construction used in [15, Proposition 2.1] to prove an analogous statement for $t \geq 0$ and then we modify it using Lemma 3 to prove the proposition.

As $z$ is expanding safe, there exist $\Delta>0, C_{0}>0$ and $\lambda>1$ so that for all $\ell \geq 1$ the map $f^{\ell}$ is univalent on $V_{\ell} \stackrel{\text { def }}{=} f_{z}^{-\ell}\left(B\left(f^{\ell}(z), \Delta\right)\right)$ and $\left|\left(f^{\ell}\right)^{\prime}(z)\right| \geq C_{0} \lambda^{\ell}$. Hence, in particular, the distortion Dist $\left.f^{\ell}\right|_{V_{\ell}}$ is bounded from above uniformly in $\ell$ by some number $C_{1}>1$. Given $r<\Delta / 2$, let $\ell=\ell(r)$ be the smallest integer satisfying $\left|\left(f^{\ell}\right)^{\prime}(z)\right| \geq C_{1} \Delta / r$. Hence, with the above, we have $f^{-\ell}\left(B\left(f^{\ell}(z), \Delta\right)\right) \subset B(z, r)$ and $\ell \leq C^{\prime \prime}-C^{\prime} \log r$, where $C^{\prime}=1 / \log \lambda$ and $C^{\prime \prime}=\left(\log \lambda+\log C_{0}^{-1} C_{1} \Delta\right) C^{\prime}$. Let $m \geq 1$ be such that $f^{m}(B(y, \Delta / 2))=J$ for any $y \in J$.

Let us choose positive constants $\alpha, \kappa$ and $n \geq m$ large enough so that $\kappa n^{-\alpha}<\Delta / 2$ and that for every $j=1, \ldots, 2 n$ for every point $z_{j} \in f^{-j}(z)$ on the component $f_{z_{j}}^{-j}\left(B\left(z, \kappa n^{-\alpha}\right)\right)$ the map $f^{j}$ is univalent and satisfies

$$
f_{z_{j}}^{-j}\left(B\left(z, \kappa n^{-\alpha}\right)\right) \subset B\left(z_{j}, \Delta / 2\right) .
$$

Note that with this choice we have for large $n$,

$$
\ell \stackrel{\text { def }}{=} \ell\left(\kappa n^{-\alpha}\right) \leq C^{\prime \prime}-C^{\prime} \log \kappa+\alpha C^{\prime} \log n \ll n-m .
$$

As $m \leq n$ and $\left.f^{m}\left(B\left(f^{\ell}(z), \Delta\right)\right)\right)$ covers $J$, we can conclude that for every preimage $z_{n} \in f^{-n}(z)$ there exists a component $W_{z_{n}}$ of $f^{-m}\left(f_{z_{n}}^{-n}\left(B\left(z, \kappa n^{-\alpha}\right)\right)\right)$ contained in $B\left(f^{\ell}(z), \Delta\right)$. The map $f^{m+n}$, and hence $f^{m+n+\ell}$, is univalent on $W_{z_{n}}$. Thus, the map

$$
F \stackrel{\text { def }}{=} f^{m+n+\ell}: \bigcup_{z_{n} \in f^{-n}(z)} W_{z_{n}} \rightarrow B\left(f^{\ell}(z), \Delta\right)
$$

has no critical points, and $Z \stackrel{\text { def }}{=} \bigcap_{k=1}^{\infty} F^{-k}\left(B\left(f^{\ell}(z), \Delta\right)\right)$ is a uniformly expanding repeller with respect to $F$.

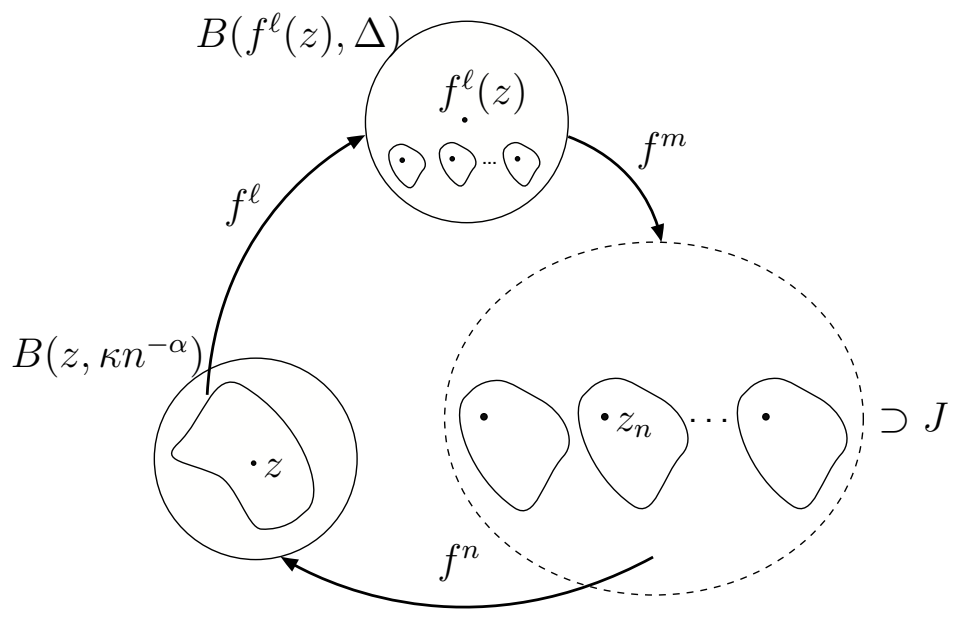

Figure 1. Construction of the uniformly expanding repeller $Z$.

Let us now slightly modify the construction of $Z$ by (12) and ignore all those backward branches $f^{-(m+n+\ell)}$ that correspond to a point $z_{n} \in V$. Given $\kappa=\Delta / 2$, let us consider the positive integers $N \leq M$ and the number $\delta>0$ provided by Lemma 3. Then, by Lemma 3, for each point $z_{n} \in f^{-n}(z) \cap V$ there exist numbers 
$j\left(z_{n}\right), i\left(z_{n}\right) \leq M$, a point $z_{n}^{*} \in f^{-\left(N+j\left(z_{n}\right)\right)}\left(f^{i\left(z_{n}\right)}\left(z_{n}\right)\right)$ in $B\left(f^{\ell}(z), \Delta / 2\right)$. Any such branch stays $\delta$-far from Crit. Note that the distortion of $f^{n+N+j\left(z_{n}\right)-i\left(z_{n}\right)+\ell}$ on

$$
W_{z_{n}}^{*} \stackrel{\text { def }}{=} f_{z_{n}^{*}}^{-\left(n+N+j\left(z_{n}\right)-i\left(z_{n}\right)\right)}\left(B\left(z, \kappa n^{-\alpha}\right)\right)
$$

is bounded by a constant $D>1$ independent of $z_{n}$ and $n$. Given an integer $k \in$ $\{n+N-M, \ldots, n+N+M\}$, put

$$
P_{k} \stackrel{\text { def }}{=}\left\{z_{n} \in f^{-n}(z) \backslash V: n+N+j\left(z_{n}\right)-i\left(z_{n}\right)=k\right\} .
$$

Note that for distinct $z_{n}$ and $z_{n}^{\prime}$ in $P_{k}$ the sets $W_{z_{n}}^{*}$ and $W_{z_{n}^{\prime}}^{*}$ are disjoint. Setting

$$
F_{k}^{*} \stackrel{\text { def }}{=} f^{k+\ell}: \bigcup_{z_{n} \in P_{k}} W_{z_{n}}^{*} \rightarrow B\left(f^{\ell}(z), \Delta\right)
$$

the sets

$$
Z_{k}^{*} \stackrel{\text { def }}{=} \bigcap_{j=1}^{\infty}\left(F_{k}^{*}\right)^{-j}\left(B\left(f^{\ell}(z), \Delta\right)\right) \text { and } X_{k}^{*} \stackrel{\text { def }}{=} \bigcup_{j=0}^{k+\ell-1} f^{j}\left(Z_{k}^{*}\right)
$$

are uniformly expanding repellers for $F_{k}^{*}$ and $f$, respectively. Both of these sets are disjoint from $\Sigma$ by construction. On the other hand there, letting

$$
L \stackrel{\text { def }}{=} \min \left\{1, \inf _{J \backslash B(\text { Crit }, \delta)}\left|f^{\prime}\right|\right\} \quad \text { and } \quad \widetilde{L} \stackrel{\text { def }}{=} \max \left\{1, \sup _{J}\left|f^{\prime}\right|\right\}
$$

we have

$$
\begin{aligned}
& P_{f \mid X_{k}^{*}}\left(-t \log \left|f^{\prime}\right|\right) \geq \frac{1}{k+\ell} P_{F_{k}^{*} \mid Z_{k}^{*}}\left(-t \log \left|F_{k}^{*}\right|\right) \\
& \geq \frac{1}{n+N+M+\ell} \log \left(D^{t} \sum_{z_{n} \in P_{k}}\left|\left(f^{k+\ell}\right)^{\prime}\left(z_{n}^{*}\right)\right|^{-t}\right) \\
& \geq \frac{1}{n+N+M+\ell} \log \left(D^{t}\left(C_{1}^{-1} C_{0} \lambda^{\ell}\right)^{-t} L^{-t(N+M)} \widetilde{L}^{t M} \sum_{z_{n} \in P_{k}}\left|\left(f^{n}\right)^{\prime}\left(z_{n}\right)\right|^{-t}\right) .
\end{aligned}
$$

Since $\bigcup_{k=n+N-M}^{n+N+M} P_{k}=f^{-n}(z) \backslash V$, there is $k$ such that

$$
\sum_{z_{n} \in P_{k}}\left|\left(f^{n}\right)^{\prime}\left(z_{n}\right)\right|^{-t} \geq \frac{1}{2 M+1} \sum_{z_{n} \in f^{-n}(z) \backslash V}\left|\left(f^{n}\right)^{\prime}\left(z_{n}\right)\right|^{-t}
$$

Hence, if we put $\widetilde{D} \stackrel{\text { def }}{=} D^{-1} C_{1}^{-1} C_{0} \lambda^{\ell} L^{N+M} \widetilde{L}^{-M}$, then

$$
P_{f \mid X_{k}^{*}}\left(-t \log \left|f^{\prime}\right|\right) \geq \frac{1}{n+N+M+\ell} \log \left(\widetilde{D}^{-t} \frac{1}{2 M+1} \sum_{z_{n} \in f^{-n}(z) \backslash V}\left|\left(f^{n}\right)^{\prime}\left(z_{n}\right)\right|^{-t}\right) .
$$

Since $N, M, \widetilde{D}$ are independent of $n$ and $\ell \leq C^{\prime \prime}-C^{\prime} \log \kappa+\alpha C^{\prime} \log n$, we obtain the desired assertion by taking a sufficiently large $n$.

We are now ready to prove one further equivalence.

Proposition 4. Given a sufficiently small neighborhood $V$ of $\Sigma$ and an expanding safe point $z \in J \backslash V$, for every $t \leq 0$ we have

$$
P_{\text {tree }}\left(z, \varphi_{t}, V\right)=\widetilde{P}_{\text {hyp }}\left(\varphi_{t}\right)=\widetilde{P}\left(\varphi_{t}\right)
$$


Proof. The second equality holds by Proposition 2.

By Proposition 3, we have $\widetilde{P}_{\text {hyp }}\left(\varphi_{t}\right) \geq P_{\text {tree }}\left(z, \varphi_{t}, V\right)$.

In view of Lemma 2 , to prove the inequality $\widetilde{P}_{\text {hyp }}\left(\varphi_{t}\right) \leq P_{\text {tree }}\left(z, \varphi_{t}, V\right)$ it is enough to show that for each expanding repeller $X$ that does not intersect $\Sigma$, there is a neighborhood $V_{0}$ of $\Sigma$ such that $P_{f \mid X}\left(\varphi_{t}\right) \leq P_{\text {tree }}\left(z, \varphi_{t}, V_{0}\right)$. Notice that for every $x \in X$ and every neighborhood $V_{0}$ of $\Sigma$ disjoint from $X$ we have,

$$
P_{f \mid X}\left(\varphi_{t}\right) \leq P_{\text {tree }}\left(x, \varphi_{t}, V_{0}\right) .
$$

This follows easily considering the contribution of the backward branches of $f^{-n}(x)$ contained in $X$ in the sum in (9).

Let $Y$ be a neighborhood of $X$ on which $\left.f\right|_{Y}$ is uniformly expanding. Thus, there is a constant $C>1$ and for every $y \in Y$ there is $x \in X$ such that for every integer $n \geq 1$ and every $x^{\prime} \in f^{-n}(x)$ there is $y^{\prime} \in f^{-n}(y)$ shadowing $x^{\prime}$ and so that

$$
C^{-1}<\left|\left(f^{n}\right)^{\prime}\left(y^{\prime}\right)\right| /\left|\left(f^{n}\right)^{\prime}\left(x^{\prime}\right)\right|<C .
$$

Hence, for each neighborhood $V_{0}$ disjoint from $Y$ we have $P_{\left.f\right|_{X}}\left(\varphi_{t}\right) \leq P_{\text {tree }}\left(y, \varphi_{t}, V_{0}\right)$.

By the eventually onto property of $f$ on $J$, we have $f^{m}(Y)=J$ for some $m \geq 1$. Fix $y \in Y \cap f^{-m}(z)$ and let $V_{0}$ neighborhood of $\Sigma$ disjoint from $Y$. Then we have

$$
\begin{aligned}
P_{m+n}\left(z, \varphi_{t}, V_{0}\right) & =\frac{1}{m+n} \log \sum_{x \in f^{-(m+n)}(z) \cap J \backslash V_{0}}\left|\left(f^{m+n}\right)^{\prime}(x)\right|^{-t} \\
& \geq \frac{1}{m+n} \log \left|\left(f^{m}\right)^{\prime}(y)\right|^{-t}+\frac{n}{m+n} P_{n}\left(y, \varphi_{t}, V_{0}\right) .
\end{aligned}
$$

This shows $P_{\text {tree }}\left(z, \varphi_{t}, V_{0}\right) \geq P_{\text {tree }}\left(y, \varphi_{t}, V_{0}\right)$ and completes the proof of the inequality $P_{\text {tree }}\left(z, \varphi_{t}, V_{0}\right) \geq \widetilde{P}_{\text {hyp }}\left(\varphi_{t}\right)$.

\section{4. $\sigma$-finite conformal measures}

Recall that $f$ is a rational map of degree at least 2. If $f$ is exceptional, then $\Sigma$ is the maximal finite and forward invariant subset of $J$ satisfying $f^{-1}(\Sigma) \backslash \Sigma \subset$ Crit. Otherwise $\Sigma=\varnothing$.

In the following proposition we adapt the classical method by Patterson and Sullivan to construct a $\left(e^{\widetilde{P}\left(\varphi_{t}\right)-\varphi_{t}}\right)$-conformal measure on $J$ for each $t<0$. For a map without a phase transition in the negative spectrum or for a map with a phase transition in the negative spectrum at some parameter $t_{-}<t$, we obtain a finite conformal measure supported on $J$, as in part 1 of Proposition 1 . For a map having a phase transition in the negative spectrum at some parameter $t_{-}>t$ this construction gives us a conformal measure outside Crit, which is finite outside each neighborhood of $\Sigma$. Recall that by part 2 of Proposition 1 , existence of phase transition implies that there does not exist a finite $\left(e^{\widetilde{P}\left(\varphi_{t}\right)-\varphi_{t}}\right)$-conformal measure for $t<t_{-}$.

Proposition 5. Let $f$ be a rational function of degree at least 2. For each $t<0$ there exists a Borel measure on $J$ that is $\left(e^{\widetilde{P}\left(\varphi_{t}\right)-\varphi_{t}}\right)$-conformal outside Crit, finite outside any neighborhood of $\Sigma$, gives zero measure to $\Sigma \cup$ Crit and whose support is equal to $J$.

Proof. As a first step we will apply the Patterson-Sullivan method while considering only those inverse branches outside a given neighborhood $V$ of $\Sigma$. We obtain in 
this way a measure that is $\left(e^{\widetilde{P}\left(\varphi_{t}\right)-\varphi_{t}}\right)$-conformal outside the set $\bar{V} \cup f^{-1}(\bar{V}) \cup$ Crit. We will obtain a measure $\left(e^{\widetilde{P}\left(\varphi_{t}\right)-\varphi_{t}}\right)$-conformal outside Crit by taking the limit of the measures obtained by repeating this construction with $V$ replaced by smaller and smaller neighborhoods.

We start with the following lemma. Recall that $\Sigma_{0}$ denotes the set of neutral periodic points in $\Sigma$ plus its preimages.

Lemma 4. Given $t<0$, for every $\lambda>0$ there exist positive numbers $r$ and $A$ such that for every $x \in J$ and every integer $\ell \geq 1$ we have

$$
\sum\left|\left(f^{\ell}\right)^{\prime}(y)\right|^{-t} \leq A e^{\ell \lambda}
$$

where the sum is taken over all $y \in f^{-\ell}(x)$ satisfying $f^{j}(y) \in B\left(\Sigma_{0}, r\right)$ for every $j \in\{0, \ldots, \ell-1\}$.

Proof. Let $r>0$ be sufficiently small so that for each periodic point $p \in \Sigma_{0}$ of minimal period $n \geq 1$ we have

$$
\sup _{y \in B(p, r)}\left|\left(f^{n}\right)^{\prime}(y)\right|^{-t} \leq e^{\lambda n} .
$$

Hence, there is some constant $A_{0}>0$ such for every integer $\ell \geq 1$ and every point $y$ satisfying $f^{j}(y) \in B\left(\Sigma_{0}, r\right)$ for every $j \in\{0, \ldots, \ell-1\}$ we have

$$
\left|\left(f^{\ell}\right)^{\prime}(y)\right|^{-t} \leq A_{0} e^{\ell \lambda} .
$$

Reducing $r$ if necessary, we may assume that for every $p \in \Sigma_{0}$ the map $f$ is injective on $B(p, r)$ and the set $f(B(p, r))$ is disjoint from $B\left(\Sigma_{0} \backslash\{f(p)\}, r\right)$. So for each $p \in \Sigma_{0}$ and $w \in B(f(p), r)$ there is at most one point $w^{\prime} \in B(p, r)$ such that $f\left(w^{\prime}\right)=w$. By induction we can conclude that for each $\ell \geq 1, x \in J$, and $p, p^{\prime} \in \Sigma_{0}$ there is at most one point $y \in f^{-\ell}(x)$ such that

$$
y \in B(p, r) \quad \text { and } \quad f^{\ell-1}(y) \in B\left(p^{\prime}, r\right) .
$$

Thus the assertion follows with $A \stackrel{\text { def }}{=} A_{0}\left(\operatorname{card} \Sigma_{0}\right)^{2}$.

We now continue in proving the proposition. Let $z \in J \backslash \Sigma$ be an expanding safe point. Given $\lambda=\widetilde{P}\left(\varphi_{t}\right) / 3$, let $r$ and $A$ be the positive numbers provided by Lemma 4. Reducing $r>0$ if necessary, we can assume that $z \notin B(\Sigma, r)$ and by Proposition 4 we can assume that $V \stackrel{\text { def }}{=} B(\Sigma, r)$ satisfies $P_{\text {tree }}\left(z, \varphi_{t}, V\right)=\widetilde{P}\left(\varphi_{t}\right)$.

There exists a sequence $\left(b_{n}\right)_{n \geq 1}$ of positive reals such that

$$
\sum_{n=1}^{\infty} b_{n} e^{-n p} \sum_{x \in f^{-n}(z) \cap J \backslash V}\left|\left(f^{n}\right)^{\prime}(x)\right|^{-t} \begin{cases}<\infty & \text { if } p>\widetilde{P}\left(\varphi_{t}\right), \\ =\infty & \text { if } p \leq \widetilde{P}\left(\varphi_{t}\right),\end{cases}
$$

and $\lim _{n \rightarrow \infty} b_{n} / b_{n+1}=1$ (see, for example, [5, Lemma 3.1]). Given $t<0$ and $p>\widetilde{P}\left(\varphi_{t}\right)$, let us define

$$
M_{t, p} \stackrel{\text { def }}{=} \sum_{n=1}^{\infty} b_{n} e^{-n p} \sum_{x \in f^{-n}(z) \cap J \backslash V}\left|\left(f^{n}\right)^{\prime}(x)\right|^{-t}
$$


and for each neighborhood $W$ of $\Sigma$ define the measure

$$
\mu_{t, W, p} \stackrel{\text { def }}{=} \frac{1}{M_{t, p}} \sum_{n=1}^{\infty} b_{n} e^{-n p} \sum_{x \in f^{-n}(z) \cap J \backslash W}\left|\left(f^{n}\right)^{\prime}(x)\right|^{-t} \delta_{x},
$$

where $\delta_{x}$ denotes the Dirac measure supported at $x$.

Observe that the measure $\mu_{t, V, p}$ is probabilistic for any $p>\widetilde{P}\left(\varphi_{t}\right)$. If $W \subset V$ is a neighborhood of $\Sigma$ the measure $\mu_{t, W, p}$ is not probabilistic in general, however it is finite as shown in the following lemma. Let us denote by $|\mu|$ the total mass of a measure $\mu$, that is, let $|\mu|=\mu(J)$.

Lemma 5. For every neighborhood $W$ of $\Sigma$ contained in $V$ there is a positive constant $C(W)$ such that for every $p>\widetilde{P}\left(\varphi_{t}\right)$ we have

$$
1 \leq\left|\mu_{t, W, p}\right| \leq C(W) \text {. }
$$

Proof. Since by assumption $W \subset V$, we have $\left|\mu_{t, W, p}\right| \geq\left|\mu_{t, V, p}\right|=1$.

It only remains to prove the upper bound. Put

$$
V_{1} \stackrel{\text { def }}{=} V \text {, and for } i \geq 1 \text { put } V_{i+1} \stackrel{\text { def }}{=} V_{i} \cap f^{-1}\left(V_{i}\right) .
$$

Note that $V_{i+1}$ is the subset of $V$ consisting of all points that under forward iteration do not leave $V$ for at least $i$ steps. In particular,

$$
f\left(V_{i} \backslash V_{i+1}\right) \subset \overline{\mathbf{C}} \backslash V_{i}
$$

for all $i \geq 1$.

We will first consider a simple case and assume that we have $V_{i_{0}} \subset W$ for some integer $i_{0} \geq 1$. We establish an upper bound for $\left|\mu_{t, V_{i_{0}}, p}\right|$ and hence for $\left|\mu_{t, W, p}\right|$. Observe first that for every $i \geq 1$ we have

$$
\left|\mu_{t, V_{i+1}, p}\right|=\mu_{t, V_{i+1}, p}\left(V_{i} \backslash V_{i+1}\right)+\mu_{t, V_{i+1}, p}\left(J \backslash V_{i}\right) \leq \mu_{t, V_{i+1}, p}\left(V_{i} \backslash V_{i+1}\right)+\left|\mu_{t, V_{i}, p}\right| .
$$

By (16) for any point $x \in V_{i} \backslash V_{i+1}$ we have $f(x) \in \overline{\mathbf{C}} \backslash V_{i}$. Hence, we can estimate

$$
\begin{aligned}
& M_{t, p} \cdot \mu_{t, V_{i+1}, p}\left(V_{i} \backslash V_{i+1}\right)=\sum_{n=1}^{\infty} b_{n} e^{-n p} \sum_{x \in f^{-n}(z) \cap V_{i} \backslash V_{i+1}}\left|\left(f^{n}\right)^{\prime}(x)\right|^{-t} \\
& \leq e^{-p} \operatorname{deg} f \sup _{J}\left|f^{\prime}\right|^{-t} \sum_{n=1}^{\infty} b_{n} e^{-(n-1) p} \sum_{y \in f^{-(n-1)}(z) \cap J \backslash V_{i}}\left|\left(f^{n-1}\right)^{\prime}(y)\right|^{-t} \\
& \leq e^{-p} \operatorname{deg} f \sup _{J}\left|f^{\prime}\right|^{-t}\left(b_{1}+\max _{k \geq 2} \frac{b_{k}}{b_{k-1}} \sum_{n=1}^{\infty} b_{n} e^{-n p} \sum_{x \in f^{-n}(z) \cap J \backslash V_{i}}\left|\left(f^{n}\right)^{\prime}(x)\right|^{-t}\right) \\
& =e^{-p} \operatorname{deg} f \sup _{J}\left|f^{\prime}\right|^{-t}\left(b_{1}+\max _{k \geq 2} \frac{b_{k}}{b_{k-1}} M_{t, p} \cdot\left|\mu_{t, V_{i}, p}\right|\right) .
\end{aligned}
$$

Since $\lim _{k \rightarrow \infty} b_{k} / b_{k+1}=1$, we have $\max _{k \geq 2} b_{k} / b_{k-1}<\infty$. So, if choosing constants $C_{0}=\operatorname{deg} f \cdot \max _{k \geq 2} b_{k} / b_{k-1}$ and $C_{1}=\operatorname{deg} f \cdot b_{1} M_{t, p}^{-1}$, we obtain

$$
\left|\mu_{t, V_{i+1}, p}\right| \leq C_{1} e^{-p} \sup _{J}\left|f^{\prime}\right|^{-t}+\left|\mu_{t, V_{i}, p}\right|\left(1+C_{0} e^{-p} \sup _{J}\left|f^{\prime}\right|^{-t}\right) .
$$


Now let $C \stackrel{\text { def }}{=} 1+C_{1} C_{0}^{-1}$ and $C^{\prime} \stackrel{\text { def }}{=} C_{0}$. Since $\left|\mu_{t, V_{1}, p}\right|=1$, we obtain by induction in $i$ that

$$
\left|\mu_{t, V_{i_{0}}, p}\right| \leq C\left(1+C^{\prime} e^{-p} \sup _{J}\left|f^{\prime}\right|^{-t}\right)^{i_{0}-1} .
$$

Finally, recalling that $\left|\mu_{t, W, p}\right| \leq\left|\mu_{t, V_{i_{0}}, p}\right|$, this proves the lemma in this first simple case that we considered.

Naturally, if $\Sigma=\Sigma_{+}$then we can choose $V$ in such a way that $\bigcap_{i=1}^{\infty} V_{i}=\Sigma$ and then it would be enough to consider the above case in which $V_{i}$ is eventually contained in $W$. However, if $\Sigma_{0} \neq \varnothing$, that is, if $\Sigma$ contains a neutral periodic point in $J$ then this is not possible by the existence of Siegel compacta [11, Theorem 1].

Let us now consider the general case. Recall that $V=B(\Sigma, r)$. Let $W \subset V$ be an arbitrary neighborhood of $\Sigma$. Certainly we can take $i \geq 1$ sufficiently large such that $V_{i} \cap B\left(\Sigma_{+}, r\right) \subset W$. Increasing $i$ if necessary, we can assume that for every integer $k \geq i$ we have $b_{k+1} / b_{k} \leq e^{\widetilde{P}\left(\varphi_{t}\right) / 3}$. For each $x \in f^{-n}(z) \cap J \backslash W$ one of the following two cases can occur: Either a) $x \notin V_{i}$ or b) $x \in V_{i} \backslash W$, and hence

$$
M_{t, p} \cdot\left|\mu_{t, W, p}\right| \leq M_{t, p} \cdot\left|\mu_{t, V_{i}, p}\right|+\sum_{n=1}^{\infty} \sum_{y \in f^{-n}(z) \cap J \cap V_{i} \backslash W} b_{n} e^{-n p}\left|\left(f^{n}\right)^{\prime}(y)\right|^{-t} .
$$

In evaluating the latter term observe that to each point $x \in f^{-n}(z) \backslash V_{i}$ we may find some branch of preimages determined by a point $y \in f^{-\ell}(x)$ satisfying $f^{j}(y) \in V_{i} \backslash W$ and hence $f^{j}(y) \in B\left(\Sigma_{0}, r\right)$ for every $j \in\{0, \ldots, \ell-1\}$. However, by Lemma 4 , given any $x \in V_{i}$ the contribution of all such branches can be estimated by

$$
\begin{aligned}
\sum_{y \in f^{-\ell}(x)} b_{n+\ell} e^{-(n+\ell) p}\left|\left(f^{n+\ell}\right)^{\prime}(y)\right|^{-t} & =b_{n} e^{-n p}\left|\left(f^{n}\right)^{\prime}(x)\right|^{-t} \cdot \frac{b_{n+\ell}}{b_{n}} e^{-\ell p} \sum_{y \in f^{-\ell}(x)}\left|\left(f^{\ell}\right)^{\prime}(y)\right|^{-t} \\
& \leq b_{n} e^{-n p}\left|\left(f^{n}\right)^{\prime}(x)\right|^{-t} \cdot e^{-\ell p} \max _{k \geq i} \frac{b_{k+\ell}}{b_{k}} A e^{\ell \lambda}
\end{aligned}
$$

where each sum is taken over all $y$ so that $f^{j}(y) \in B\left(\Sigma_{0}, r\right)$ for every $j \in\{0, \ldots, \ell-1\}$. Thus, summing over all such branches that could occur, by our previous choice of $i$ and $\lambda$ we can estimate

$$
\begin{aligned}
\left|\mu_{t, W, p}\right| & \leq\left|\mu_{t, V_{i}, p}\right|\left(1+\sum_{\ell=1}^{\infty}\left(e^{-\ell p} \max _{k \geq i}\left(\frac{b_{k+1}}{b_{k}}\right)^{\ell} A e^{\ell \lambda}\right)\right) \\
& \leq\left|\mu_{t, V_{i}, p}\right|\left(1+A \sum_{\ell=1}^{\infty} e^{-\ell p} e^{2 \ell \widetilde{P}\left(\varphi_{t}\right) / 3}\right) \leq\left|\mu_{t, V_{i}, p}\right|\left(1+A \sum_{\ell=1}^{\infty} e^{-\ell \widetilde{P}\left(\varphi_{t}\right) / 3}\right) .
\end{aligned}
$$

Note that $\widetilde{P}\left(\varphi_{t}\right)>0$. Together with (17) this completes the proof of the lemma.

Lemma 6. Given a neighborhood $W$ of $\Sigma$ contained in $V$, as $p \searrow \widetilde{P}\left(\varphi_{t}\right)$ there exists a non-zero finite measure that is a weak* accumulation point of the family of measures $\left\{\mu_{t, W, p}: p>\widetilde{P}\left(\varphi_{t}\right)\right\}$. Furthermore, each such measure is $\left(e^{\widetilde{P}\left(\varphi_{t}\right)-\varphi_{t}}\right)$ conformal outside the set $\bar{W} \cup f^{-1}(\bar{W}) \cup$ Crit.

Proof. First observe that, by Lemma 5 the total mass of any of the measures in $\left\{\mu_{t, V_{i}, p}: p>\widetilde{P}\left(\varphi_{t}\right)\right\}$ is uniformly bounded from above and below by some positive 
constant. Hence this family of measures is relatively compact in the weak* topology and thus possesses a non-zero and finite accumulation point proving the first claim.

Following the construction in [5, Section 3], we have for every special set $A$ disjoint from $W \cup f^{-1}(W) \cup$ Crit

$$
\begin{aligned}
\mu_{t, W, p}(f(A)) & =\frac{1}{M_{t, p}} \sum_{n=1}^{\infty} \sum_{y \in f(A) \cap f^{-n}(z)} b_{n} e^{S_{n} \varphi_{t}(y)-n p} \\
& =\frac{1}{M_{t, p}} \sum_{n=1}^{\infty} \sum_{x \in A \cap f^{-(n+1)}(z)} b_{n} e^{S_{n} \varphi_{t}(f(x))-n p} \\
& =\frac{1}{M_{t, p}} \sum_{n=1}^{\infty} \sum_{x \in A \cap f^{-(n+1)}(z)} b_{n} e^{S_{n+1} \varphi_{t}(x)-(n+1) p} e^{p-\varphi_{t}(x)}
\end{aligned}
$$

Thus,

$$
\begin{aligned}
& \Delta_{A}(t, W, p) \stackrel{\text { def }}{=}\left|\mu_{t, W, p}(f(A))-\int_{A} e^{\widetilde{P}\left(\varphi_{t}\right)-\varphi_{t}} d \mu_{t, W, p}\right| \\
& =\frac{1}{M_{t, p}} \mid \sum_{n=1}^{\infty} \sum_{x \in A \cap f^{-(n+1)}(z)} e^{S_{n+1} \varphi_{t}(x)-(n+1) p} e^{-\varphi_{t}(x)}\left[b_{n} e^{p}-b_{n+1} e^{\widetilde{P}\left(\varphi_{t}\right)}\right] \\
& \quad-b_{1} \sum_{x \in A \cap f^{-1}(z)} e^{\widetilde{P}\left(\varphi_{t}\right)-p} \mid \\
& \leq \frac{1}{M_{t, p}} \sum_{n=1}^{\infty} \sum_{x \in A \cap f^{-(n+1)}(z)} b_{n+1}\left|\frac{b_{n}}{b_{n+1}}-e^{\widetilde{P}\left(\varphi_{t}\right)-p}\right| e^{p-\varphi_{t}(x)} e^{S_{n+1} \varphi_{t}(x)-(n+1) p} \\
& \quad+\frac{1}{M_{t, p}} b_{1} \operatorname{deg} f \cdot e^{\widetilde{P}\left(\varphi_{t}\right)-p} .
\end{aligned}
$$

Recall that, by the choice of $\left(b_{n}\right)_{n \geq 1}$ in (13), we have $\lim _{n \rightarrow \infty} b_{n} / b_{n+1}=1$ and $\lim _{p \searrow \widetilde{P}\left(\varphi_{t}\right)} M_{t, p}=\infty$. Hence, we obtain $\lim _{p \searrow \widetilde{P}\left(\varphi_{t}\right)} \Delta_{A}(t, W, p)=0$ uniformly in $A$. The assertion now follows like in [5, Section 3] (see also Section 12.1 or Lemma 12.5.5 and Remark 12.5.6 in [16]). This proves the lemma.

We are now prepared to finish the proof of the proposition. Note that in (14) we use the same normalization factor $M_{t, p}$ for all measures $\mu_{t, W, p}$ for any neighborhood $W$. Hence given $p>\widetilde{P}\left(\varphi_{t}\right)$ for any pair of neighborhoods $W$ and $W^{\prime}$ of $\Sigma$ such that $W^{\prime} \subset W \subset V$ we have

$$
\left.\mu_{t, W^{\prime}, p}\right|_{J \backslash \bar{W}}=\left.\mu_{t, W, p}\right|_{J \backslash \bar{W}}
$$

Using a diagonal argument we can conclude that, as $p \searrow \widetilde{P}\left(\varphi_{t}\right)$ and $\rho \rightarrow 0$, there exists a weak* accumulation measure $\nu_{t}$ of the family

$$
\left\{\mu_{t, B(\Sigma, \varepsilon), p}: t>\widetilde{P}\left(\varphi_{t}\right), \varepsilon \in(0, r)\right\}
$$

and that $\nu_{t}$ is $\left(e^{\widetilde{P}\left(\varphi_{t}\right)-\varphi_{t}}\right)$-conformal outside $\Sigma \cup$ Crit. Replacing the measure $\nu_{t}$ by the restricted measure $\left.\nu_{t}\right|_{J \backslash(\Sigma \cup \text { Crit })}$, if necessary we can assume that $\nu_{t}$ does not give weight to $\Sigma \cup$ Crit and hence that $\nu_{t}$ is conformal outside Crit. 
Lemma 5 and (19) together imply that then $\nu_{t}$ is finite outside each neighborhood of $\Sigma$.

Finally, the fact that the support of $\nu_{t}$ is equal to $J$ follows from the property that $f$ is locally eventually onto on $J$. This finishes the proof of Proposition 5.

\section{Proof of the main result}

In this section we prove Theorem 1. In Section 5.1 we make use of the "bridges construction" in [7] to prove the lower bound for dimension in (6) and (7), in the statement of Theorem 1, that follows along the same lines as in [7, Sections 2.2, 2.3, and 5]. We point out that, after a careful observation, in fact it applies without any changes to our present more general setting. In Section 5.1 we also give another application of the bridges construction (Lemma 8 and its Corollary 1), that is used in Section 5.3. The upper bound in (6) and (7) is shown in Section 5.2, where in the case $t<0$ we use the $\sigma$-conformal measure given by Proposition 5 . In Section 5.3 we give the last ingredient of the proof of Theorem 1, that the upper Lyapunov exponent of each point in $J \backslash \Sigma$ is at most $\widetilde{\alpha}^{+}$(Proposition 10). The proof of Theorem 1 is given at the end of Section 5.3.

5.1. Lower bound. We refer the reader to [7] for all the notation in this subsection.

We call a point $x \in J$ non-immediately postcritical if there exists some preimage branch $x_{0}=x=f\left(x_{1}\right), x_{1}=f\left(x_{2}\right), \ldots$ that is dense in $J$ and disjoint from Crit. There are at most finitely many non-immediately postcritical points. On the other hand, it is easy to see that each periodic point not in $\Sigma$ is non-immediately postcritical. It follows that every forward invariant set disjoint from $\Sigma$ contains at least one non-immediately postcritical point.

A set $\Lambda$ is called $f$-uniformly expanding Cantor repeller (ECR) if it is a uniformly expanding repeller and limit set of a finite graph directed system (GDS) satisfying the strong separation condition (SSC) with respect to $f$. To be more precise, a GDS satisfying SSC has the following properties:

(i) There exists a finite family $\mathcal{U}=\left\{U_{k}: k=1, \ldots, K\right\}$ of open connected (not necessarily simply connected) domains with pairwise disjoint closures;

(ii) There exists a family $G=\left\{g_{k \ell}: k, \ell \in\{1, \ldots, K\}\right\}$ of branches of $f^{-1}$ mapping $\overline{U_{\ell}}$ into $U_{k}$ with bounded distortion (unlike in a general definition, here we assume that there is at most one branch for each pair $(k, \ell)$ );

(iii) If we put $D \stackrel{\text { def }}{=} \bigcup_{k=1}^{K} U_{k}$, then we have

$$
\Lambda=\bigcap_{n=1}^{\infty} \bigcup_{k_{1}, \ldots, k_{n}} g_{k_{1} k_{2}} \circ g_{k_{2} k_{3}} \circ \cdots \circ g_{k_{n-1} k_{n}}(\bar{D}),
$$

(in fact, by (ii), we can omit closure and replace $\bar{D}$ by $D$ in this formula). We assume that $f(\Lambda)=\Lambda$ and hence that for each $k$ there exists $\ell$ and for each $\ell$ there exists $k$ such that $g_{k \ell} \in G$.

We can view $k=1, \ldots, K$ (or the elements of $\mathcal{U}$ ) as vertices and $g_{k \ell}$ as edges of a directed graph $\Gamma=\Gamma(\mathcal{U}, G)$. By construction, $f$ is uniformly expanding on the limit set $\Lambda$. By condition (iii), the directed graph is transitive and hence $\left.f\right|_{\Lambda}$ is topologically transitive. 
The proof of the following lemma is based on a construction of "bridges" between two ECR's. For completeness, we will sketch its proof.

Lemma 7. [7, Lemma 2] For any two disjoint $f$-ECR sets $\Lambda_{1}, \Lambda_{2} \subset J$ that both contain non-immediately postcritical points there exists an $f$-ECR set $\Lambda \subset J \backslash \Sigma$ containing the set $\Lambda_{1} \cup \Lambda_{2}$. If $f$ is topologically transitive on each $\Lambda_{i}, i=1,2$, then $f$ is topologically transitive on $\Lambda$.

Sketch of Proof. Considering the GDS's $\mathcal{U}_{i}$ with coverings $D_{i}=\bigcup_{k=1}^{K_{i}} U_{i, k}$, we choose two corresponding non-immediately postcritical points $p_{i} \in \Lambda_{i}, i=1,2$. A choice of backward trajectories $y_{i, 0}=p_{i}, f\left(y_{i, 1}\right)=y_{i, 0}, \ldots, y_{i, t_{i}}$ of $p_{i}$ satisfying $y_{i, t} \notin \overline{D_{1}} \cup \overline{D_{2}}$ for every $t=1, \ldots, t_{i}-1, i=1,2$, and $y_{1, t_{1}} \in D_{2}, y_{2, t_{2}} \in D_{1}$ defines two "bridges" between $\Lambda_{1}$ and $\Lambda_{2}$. Further, we choose sufficiently small open discs $V_{i} \subset D_{i}$ containing $p_{i}$ and having the property that their preimages along the bridge $y_{i, 1}, \ldots, y_{i, t_{i}-1}$ are all disjoint with $\overline{D_{1}} \cup \overline{D_{2}}, i=1,2$, and their preimages at $y_{i, t_{i}}$ are in $D_{j}$ for $i \neq j, i=1,2$. Finally, we choose some sufficiently large integer $N$ such that the component of $f_{p_{i}}^{-N}\left(D_{i}\right)$ which contains $p_{i}$ is contained in $V_{i}$.

Consider the directed graph $\Gamma$ composed of the vertices numbering the domains of the family $G_{i}^{N}\left(\mathcal{U}_{i}\right)$ for $N$ large enough so that the component $\widehat{V}_{i} \in G_{i}^{N}\left(U_{i, k}\right)$ containing $p_{i}$ is contained in $V_{i}$, and of the family of the $f^{t}$-preimages of $\widehat{V}_{i}, t=$ $1, \ldots, t_{i}+N-1$, along the corresponding bridges to $\Lambda_{j}$ prolonged by $G_{j}$ 's, $j \neq i, i=$ 1,2 , and of the edges in (ii) being the branches of $f^{-1}$ involved in this construction. This defines a GDS satisfying the SSC (after a minor modification of the domains to "telescope").

If $f$ is topologically transitive on each $\Lambda_{i}$, then the corresponding directed graphs $\Gamma_{i}$ are strongly connected (i.e. each two vertices are joined by a path of directed edges). Then, by construction, $\Gamma$ is also strongly connected. Hence $f$ is topologically transitive on $\Lambda$.

The following proposition generalizes [7, Proposition 1].

Proposition 6. There exists a sequence $\left(a_{m}\right)_{m \geq 1}$ of positive integers and a sequence $\left(\Lambda_{m}\right)_{m \geq 1}$ of subsets of $J \backslash \Sigma$, such that for each $m$ the set $\Lambda_{m}$ is $f^{a_{m}}$-invariant and uniformly expanding topologically transitive set, in such a way that for every $t \in \mathbf{R}$ we have

$$
\widetilde{P}\left(\varphi_{t}\right)=\lim _{m \rightarrow \infty} \frac{1}{a_{m}} P_{f^{a_{m} \mid \Lambda_{m}}}\left(S_{a_{m}} \varphi_{t}\right)=\sup _{m \geq 1} \frac{1}{a_{m}} P_{f^{a_{m} \mid \Lambda_{m}}}\left(S_{a_{m}} \varphi_{t}\right) .
$$

Proof. Recall the definition of the hidden hyperbolic pressure $\widetilde{P}_{\text {hyp }}\left(\varphi_{t}\right)$ in (8). By Propositions 2 and 4 this pressure coincides with $\widetilde{P}\left(\varphi_{t}\right)$ and is obtained by taking a supremum over uniformly expanding repellers. Note that, given $t \in \mathbf{R}$ and $\varepsilon>0$ and a uniformly expanding repeller $\Lambda$, by [7, Lemma 3] there exists a positive integer $n$ and an $f^{n}$-ECR $\Lambda^{\prime} \subset \Lambda$ such that

$$
\frac{1}{n} P_{f^{n} \mid \Lambda^{\prime}}\left(S_{n} \varphi_{t}\right) \geq P_{f \mid \Lambda}\left(\varphi_{t}\right)-\varepsilon
$$

Note that in this case $\Lambda^{\prime} \cap \Sigma=\varnothing$ since $\Lambda \cap \Sigma=\varnothing$. Hence $\Lambda^{\prime}$ contains non-immediately postcritical points. Note further that, given any two $f$-ECR's $\Lambda_{1}$ and $\Lambda_{2}$ that both contain non-immediately postcritical points, by Lemma 7 there exists an $f$-ECR 
$\Lambda \subset J \backslash \Sigma$ containing $\Lambda_{1} \cup \Lambda_{2}$ and thus with pressure at least equal to the maximum of pressures of $\Lambda_{1}$ and $\Lambda_{2}$.

Based on these arguments, we can conclude that for any $N>0$ and $\varepsilon>0$ we can find an integer $n \geq 1$ and a topologically transitive $f^{n}$-ECR $\Lambda \subset J$ so that

$$
\frac{1}{n} P_{f^{n} \mid \Lambda}\left(\varphi_{t}\right) \geq \widetilde{P}\left(\varphi_{t}\right)-\varepsilon
$$

for all $t \in(-N, N)$. This proves the proposition.

The existence of such an approximating sequence of repellers and [7, Theorem 3] together imply the following estimate, that is part of Theorem 1.

Proposition 7. For $\alpha^{-} \leq \alpha \leq \beta \leq \widetilde{\alpha}^{+}$we have

$$
\operatorname{dim}_{\mathrm{H}} \mathcal{L}(\alpha, \beta) \geq \min \{\widetilde{F}(\alpha), \widetilde{F}(\beta)\}
$$

Proof. Consider now a sequence $\left(\Lambda_{m}\right)_{m \geq 1}$ of $f^{a_{m}}$-ECRs as provided by Proposition 6 and assume that the spectrum of Lyapunov exponents of $\Lambda_{m}$ eventually contains any exponent in $\left(\alpha^{-}, \widetilde{\alpha}^{+}\right)$. Given $\alpha, \beta \in\left[\alpha^{-}, \widetilde{\alpha}^{+}\right]$we can choose a sequence $\left(\gamma_{m}\right)_{m \geq 1}$ so that $\liminf _{m \rightarrow \infty} \gamma_{m}=\alpha$ and $\lim \sup _{m \rightarrow \infty} \gamma_{m}=\beta$ and that each $\gamma_{m}$ is a Lyapunov exponent of $\left.f^{a_{m}}\right|_{\Lambda_{m}}$. Thus, for each $m \geq 1$ there exist a unique number $t_{m}=t_{m}\left(\gamma_{m}\right) \in \mathbf{R}$ so that $a_{m} \gamma_{m}=-\left.\frac{d}{d s} P_{f^{a_{m} \mid \Lambda_{m}}}\left(S_{a_{m}} \varphi_{s}\right)\right|_{s=t_{m}}$. Moreover, there exists an equilibrium state $\mu_{m}$ for the potential $S_{a_{m}} \varphi_{t_{m}}$ with respect to $\left.f^{a_{m}}\right|_{\Lambda_{m}}$ with Lyapunov exponent (with respect to $f^{a_{m}}$ ) equal to $a_{m} \gamma_{m}$ and satisfying

$$
\begin{aligned}
\operatorname{dim}_{\mathrm{H}} \mu_{m} & =\frac{h_{\mu_{m}}\left(f^{a_{m}}\right)}{a_{m} \chi\left(\mu_{m}\right)}=\frac{P_{f^{a} \mid \Lambda_{m}}\left(\varphi_{t_{m}}\right)+t_{m} a_{m} \gamma_{m}}{a_{m} \gamma_{m}} \\
& \geq \frac{1}{a_{m} \gamma_{m}} \inf _{t \in \mathbf{R}}\left(P_{f^{a_{m} \mid \Lambda_{m}}}\left(\varphi_{t}\right)+t a_{m} \gamma_{m}\right) \stackrel{\text { def }}{=} F_{f^{a_{m} \mid \Lambda_{m}}}\left(\gamma_{m}\right) .
\end{aligned}
$$

By Proposition 6 and (5), we can conclude that $F_{f^{a m_{k} \mid \Lambda_{m_{k}}}}\left(\gamma_{m_{k}}\right) \rightarrow \widetilde{F}(\alpha)$ if $\gamma_{m_{k}} \rightarrow \alpha$ and $F_{f^{a} m_{k} \mid \Lambda_{m_{k}}}\left(\gamma_{m_{k}}\right) \rightarrow \widetilde{F}(\beta)$ if $\gamma_{m_{k}} \rightarrow \beta$. Together with [7, Theorem 3], this proves the proposition.

There is one more useful application of the bridges construction.

Lemma 8. Given a repelling periodic point $p \notin \Sigma$ and $\varepsilon>0$, there exist a uniformly expanding repeller $\Lambda$ disjoint with $\Sigma$ of positive Hausdorff dimension, containing $p$, and an ergodic nonatomic measure $\mu$ supported on $\Lambda$ such that

$$
|\chi(\mu)-\chi(p)|<\varepsilon .
$$

Proof. We start from the orbit $P$ of the periodic point $p$. As $p \notin \Sigma, p$ is nonimmediately postcritical. Hence, we can find a bridge from $P$ going back to $P$ and construct $\Lambda$ as in Lemma 7. We can then distribute a Gibbs measure on $\Lambda$ choosing potential in such a way that probability of the backward branch going through the bridge is very small.

Corollary 1. In the definition of $\widetilde{\alpha}^{+}$, instead of nonatomic ergodic measures one can use ergodic measures with support outside $\Sigma$ or ergodic measures giving measure zero to $\Sigma$.

5.2. Upper bound. Recall that a point $x$ is called conical if there exists a number $r(x)>0$, a sequence of numbers $n_{\ell}=n_{\ell}(x) \nearrow \infty$, and a sequence 
$U_{\ell}=U_{\ell}(x)$ of neighborhoods of $x$ such that $f^{n_{\ell}}\left(U_{\ell}\right)=B\left(f^{n_{\ell}}(x), r\right)$, the map $f^{n_{\ell}}$ is univalent on $U_{\ell}$ and that distortion Dist $\left.f^{n_{\ell}}\right|_{U_{\ell}}$ is bounded uniformly in $\ell$ and $x$ by a constant $K>1$ (the latter condition follows from the former one from Koebe's distortion lemma by replacing $r$ by say $r / 2$ ).

The following proposition will allow us to restrict our considerations concerning dimension to conical points with positive exponents.

Proposition 8. [7, Proposition 3] The set of points $x \in J$ that are not conical and satisfy $\bar{\chi}(x)>0$ has Hausdorff dimension zero.

We are now ready to prove an upper bound for the dimension.

Proposition 9. Let $0<\alpha \leq \beta \leq \widetilde{\alpha}^{+}$. We have

$$
\operatorname{dim}_{\mathrm{H}}\left(\bigcup_{\alpha^{\prime}, \beta^{\prime}: \alpha \leq \alpha^{\prime} \leq \beta^{\prime} \leq \beta} \mathcal{L}\left(\alpha^{\prime}, \beta^{\prime}\right)\right) \leq \max \left\{0, \max _{\alpha \leq q \leq \beta} \widetilde{F}(q)\right\} .
$$

Proof. The proof will follow the same ideas as the proof of [7, Proposition 2]. The only difference is that in the case $f$ has a phase transition in the negative spectrum and $t<t_{-}$, we will use a $\sigma$-finite conformal measure constructed in Section 4 instead of a conformal probability measure.

By Proposition 8 it is sufficient to study the subset $\mathcal{L}_{\mathrm{c}}(\alpha, \beta) \subset \mathcal{L}(\alpha, \beta)$ of points that are conical. Recall that, by the Frostman Lemma, see for example [16, Theorem 8.6.3], if there exist a finite Borel measure $\mu$ and a number $\theta$ such that for every $x \in \mathcal{L}_{\mathrm{c}}(\alpha, \beta)$ we have

$$
\underline{d}_{\mu}(x) \stackrel{\text { def }}{=} \liminf _{\delta \rightarrow 0} \frac{\log \mu(B(x, \delta))}{\log \delta} \leq \theta
$$

then $\operatorname{dim}_{\mathrm{H}} \mathcal{L}_{\mathrm{c}}(\alpha, \beta) \leq \theta$, see also [12, Theorem 7.2].

Given a point $x$ in $\mathcal{L}_{c}(\alpha, \beta) \backslash \Sigma$, there exist numbers $q(x) \in[\alpha, \beta], r(x)>0$, and $K(x)>1$, and a sequence of numbers $n_{\ell}=n_{\ell}(x)$ such that

$$
\lim _{\ell \rightarrow \infty} \frac{1}{n_{\ell}} \log \left|\left(f^{n_{\ell}}\right)^{\prime}(x)\right|=q(x)
$$

and that

$$
r\left|\left(f^{n_{\ell}}\right)^{\prime}(x)\right|^{-1} K(x)^{-1} \leq \operatorname{diam} f_{x}^{-n_{\ell}}\left(B\left(f^{n_{\ell}}(x), r\right)\right) \leq r\left|\left(f^{n_{\ell}}\right)^{\prime}(x)\right|^{-1} K(x)
$$

for all $\ell$ and all $r \in(0, r(x))$ (compare, for example, [7, Lemma 7]). By omitting finitely many $n_{\ell}$ we can assume that the right hand side of (21) is not greater than $r$. Replacing $n_{\ell}$ by $n_{\ell}-1$ and $r(x)$ by $r(x) / \sup \left|f^{\prime}\right|$ if necessary, we can also freely assume that $B\left(f^{n_{\ell}}(x), r(x)\right) \cap$ Crit $=\varnothing$.

Given $x \in \mathcal{L}_{\mathrm{c}}(\alpha, \beta)$, let us fix $r>0$ satisfying

$$
r=\frac{1}{2} \min \{r(x), \operatorname{dist}(x, \operatorname{Crit} \cup \Sigma)\} .
$$

Denote

$$
U_{\ell} \stackrel{\text { def }}{=} f_{x}^{-n_{\ell}}\left(B\left(f^{n_{\ell}}(x), r\right)\right) .
$$

Observe that $B\left(f^{n_{\ell}}(x), 2 r\right)$ does not intersect $\Sigma \cup$ Crit. Indeed, by assumption it does not intersect Crit. Further, if it intersected $\Sigma$ then either $f^{n}\left(f_{x}^{-n_{\ell}}\left(B\left(f^{n_{\ell}}(x), 2 r\right)\right)\right)$ would intersect Crit for some $0 \leq n<n_{\ell}$ or $f_{x}^{-n_{\ell}}\left(B\left(f^{n_{\ell}}(x), 2 r\right)\right)$ would intersect $\Sigma$. The former is impossible because the map would not be univalent there 
(we remind that $2 r<r(x)$ ), the latter is impossible because $\operatorname{dist}(x, \Sigma)>2 r>$ $\operatorname{diam} f_{x}^{-n_{\ell}}\left(B\left(f^{n_{\ell}}(x), 2 r\right)\right)$.

Given $t \in \mathbf{R}$ let $\mu_{t}$ be the $\sigma$-finite measure that is $\left(e^{\widetilde{P}\left(\varphi_{t}\right)-\varphi_{t}}\right)$-conformal outside Crit as provided by Proposition 5 and if $t \geq 0$ then let $\mu_{t}$ be the finite $\left(e^{\widetilde{P}\left(\varphi_{t}\right)-\varphi_{t}}\right)$ conformal measure provided by Proposition 1. Since in all cases $\mu_{t}$ is finite outside every neighborhood of $\Sigma$, if we put $\kappa=\mu_{t}(J \backslash B(\Sigma, r))$ then $\kappa$ is finite and for every $\ell$ we have,

$$
\mu_{t}\left(U_{\ell}\right) \leq \kappa K^{t} e^{-n_{\ell} \widetilde{P}\left(\varphi_{t}\right)}\left|\left(f^{n_{\ell}}\right)^{\prime}(x)\right|^{-t} .
$$

As $x$ is conical, we have

$$
U_{\ell} \supset B\left(x, K^{-1} r\left|\left(f^{n_{\ell}}\right)^{\prime}(x)\right|^{-1}\right) .
$$

Together with (22) and (20) this yields

$$
\begin{aligned}
\underline{d}_{\mu_{t}}(x) & =\liminf _{\delta \rightarrow 0} \frac{\log \mu_{t}(B(x, \delta))}{\log \delta} \\
& \leq \liminf _{\ell \rightarrow 0} \frac{\log \mu_{t}\left(B\left(x, K^{-1} r\left|\left(f^{n_{\ell}}\right)^{\prime}(x)\right|^{-1}\right)\right.}{\log \left(K^{-1} r\left|\left(f^{n_{\ell}}\right)^{\prime}(x)\right|^{-1}\right)} \leq \frac{\widetilde{P}\left(\varphi_{t}\right)+t q(x)}{q(x)} .
\end{aligned}
$$

Recall that this is true for every $x \in \mathcal{L}_{\mathrm{c}}(\alpha, \beta)$ and $t \in \mathbf{R}$. Now concluding as in the proof of [7, Proposition 2], using the Frostman lemma, we obtain that $\operatorname{dim}_{\mathrm{H}} \mathcal{L}_{\mathrm{c}}(\alpha, \beta) \leq$ $\max \left\{0, \max _{\alpha \leq q \leq \beta} \widetilde{F}(q)\right\}$, as wanted.

5.3. Completeness of the spectrum. The purpose of this section is to establish the following gap in the spectrum of upper exponents, which is the last ingredient in the proof of Theorem 1 . The proof of Theorem 1 is given at the end of this section.

Proposition 10. If $f$ is exceptional then $\bar{\chi}(x) \leq \widetilde{\alpha}^{+}$for every $x \in J \backslash \Sigma$.

We give two proofs of this proposition, one in this section and the other one in Appendix A.

Recall that we denote the spherical distance on $\overline{\mathbf{C}}$ by dist, and that for a rational map $g$ and a critical point $c \in \overline{\mathbf{C}}$ of $g$ we denote by $\operatorname{deg}_{g}(c)$ the local degree of $g$ at $z=c$.

By Corollary 1 we have $\widetilde{\alpha}^{+}<\alpha^{+}$if and only if there is a periodic point in $\Sigma$ whose exponent is strictly larger than $\widetilde{\alpha}^{+}$. Hence, to prove Proposition 10 we need to control the exponent of any point $x \in J \backslash \Sigma$ whose orbit stays most of the time close to $\Sigma$. Any orbit piece that shadows some (periodic) orbit in $\Sigma$ for a long time inherits its exponent, however right before it must have passed close to some critical point which results in a drop of the exponent.

Let us make this more precise. For $c \in f^{-1}(\Sigma) \backslash \Sigma \subset$ Crit let $k \geq 1$ be the minimal integer such that $f^{k}(c)$ is a periodic point, put

$$
\chi_{\text {ess }}(c) \stackrel{\text { def }}{=} \frac{\chi(p)}{\operatorname{deg}_{f^{k}}(c)} .
$$

and if $f$ is exceptional then we put

$$
\chi_{\mathrm{ess}}^{+} \stackrel{\text { def }}{=} \max _{c \in f^{-1}(\Sigma) \backslash \Sigma} \chi_{\mathrm{ess}}(c) .
$$


We remark that there are examples where there is a point $c \in$ Crit so that $f^{k}(c) \in \Sigma$ for some minimal number $k>1$. If $f^{k}(c) \in \Sigma_{0}$ then $\chi_{\text {ess }}(c)=0$. If $f^{k}(c) \in \Sigma_{+}$then $c^{\prime}=f^{k-1}(c)$ is a critical point in $f^{-1}(\Sigma)$ and $\chi_{\text {ess }}(c)<\chi_{\text {ess }}\left(c^{\prime}\right)$. Thus, in none of these cases the "essential exponent" of $c$ and hence of an orbit piece that would shadow some periodic orbit $\left\{f^{j}(c), j \geq k\right\}$ in $\Sigma$ would have large exponent. Hence, in what follows we can restrict ourselves to the case that $k=1$.

We have the following result.

Lemma 9. Suppose $f$ is exceptional. Let $c \in f^{-1}\left(\Sigma_{+}\right) \backslash \Sigma_{+}$and let $k \geq 1$ be the minimal integer such that $f^{k}(c) \in \Sigma_{+}$is periodic. Then there exist constants $\delta>$ 0 and $C>0$ such that for every $x \in J$ near $c$, but different from $c$, and every integer $n \geq k$ such that for every $j \in\{k, k+1, \ldots, n-1\}$ we have $f^{j}(x) \in B(\Sigma, \delta)$, the following estimate holds

$$
\log \left|\left(f^{n}\right)^{\prime}(x)\right| \leq n \chi_{\text {ess }}(c)+C .
$$

Proof. Put $d \stackrel{\text { def }}{=} \operatorname{deg}_{f^{k}}(c)$. To prove the lemma, it suffices to notice that if $\delta$ is sufficiently small then for any orbit piece $y, f(y), \ldots, f^{m}(y)$ that stays $\delta$-close to the periodic orbit of $p=f^{k}(c)$ we have $\left|\left(f^{m}\right)^{\prime}(y)\right| \sim e^{m \chi(p)}=e^{m d \chi_{\text {ess }}(c)}$. Thus

$$
\operatorname{dist}\left(f^{k}(x), f^{k}(c)\right)=\mathcal{O}\left(\left|\left(f^{n-k}\right)^{\prime}(p)\right|^{-1}\right)=\mathcal{O}\left(e^{-n d \chi_{\mathrm{ess}}(c)}\right) .
$$

On the other hand, $\operatorname{dist}(x, c) \sim \operatorname{dist}\left(f^{k}(x), f^{k}(c)\right)^{1 / d}$, so

$$
\left|\left(f^{k}\right)^{\prime}(x)\right| \sim \operatorname{dist}\left(f^{k}(x), f^{k}(c)\right)^{(d-1) / d}=\mathcal{O}\left(e^{-n(d-1) \chi_{\mathrm{ess}}(c)}\right),
$$

and $\left|\left(f^{n}\right)^{\prime}(x)\right|=\mathcal{O}\left(e^{n \chi_{\text {ess }}(c)}\right)$.

Given a subset $V$ of $\overline{\mathbf{C}}$, let

$$
\chi^{+}(J \backslash V) \stackrel{\text { def }}{=} \limsup _{n \rightarrow \infty} \sup _{x \in J \backslash V} \frac{1}{n} \log \left|\left(f^{n}\right)^{\prime}(x)\right| .
$$

Lemma 10. If $f$ is non-exceptional then there is an ergodic measure $\mu$ supported on $J$ and such that $\chi(\mu)=\chi^{+}(J)$. If $f$ is exceptional and $V$ is a neighborhood of $\Sigma$, then one of the following cases holds:

1. Either there exists an invariant ergodic measure $\mu$ such that

$$
\mu(\Sigma)=0 \quad \text { and } \quad \chi(\mu) \geq \chi^{+}(J \backslash V) ;
$$

2. $\mathrm{Or}$

$$
\chi^{+}(J \backslash V) \leq \chi_{\mathrm{ess}}^{+} .
$$

Proof. Let $V$ be empty if $f$ is not exceptional and let $V$ be a neighborhood of $\Sigma$ otherwise. Without loss of generality in the latter case we can assume that $V$ is open. Let $\delta>0$ be given by Lemma 9 . For each $n \geq 1$ let $x_{n} \in J \backslash V$ be a point satisfying

$$
\frac{1}{n} \log \left|\left(f^{n}\right)^{\prime}\left(x_{n}\right)\right|=\sup _{x \in J \backslash V} \frac{1}{n} \log \left|\left(f^{n}\right)^{\prime}(x)\right|
$$

and consider the probability measure

$$
\mu_{n} \stackrel{\text { def }}{=} \frac{1}{n} \sum_{k=0}^{n-1} \delta_{f^{k}\left(x_{n}\right)} .
$$

Consider a measure $\mu$ that is accumulated by the sequence of measures $\left(\mu_{n}\right)_{n \geq 1}$ in the weak* topology. Notice that $\mu$ is $f$-invariant and satisfies $\chi(\mu) \geq \chi^{+}(J \backslash V)$. It 
follows that there is a $f$-invariant an ergodic measure $\mu^{\prime}$ such that $\chi\left(\mu^{\prime}\right) \geq \chi^{+}(J \backslash V)$. If $f$ is not exceptional or if $f$ is exceptional and $\mu^{\prime}(\Sigma)=0$, then we are done.

To prove the remaining case, assume that $f$ is exceptional and $\operatorname{supp} \mu^{\prime} \subset \Sigma$. Fix $\varepsilon>0$ and let $\delta>0$ and $C>0$ be given by Lemma 9. Augmenting $C>0$ and reducing $\delta$ if necessary we can assume that $B(\Sigma, \delta) \subset V$ and that for each $x \in J$ and each integer $\ell \geq 1$ so that $f^{j}(x) \in B\left(\Sigma_{0}, \delta\right)$ for every $j \in\{0, \ldots, \ell-1\}$, we have

$$
\left|\left(f^{\ell}\right)^{\prime}(x)\right| \leq e^{\ell \varepsilon+C} \text {. }
$$

Let $V_{+}$be a neighborhood of $\Sigma_{+}$that is contained in $B\left(\Sigma_{+}, \delta\right)$ and such that all preimages of a point in $V_{+}$are either contained in $V_{+}$or close to $f^{-1}\left(\Sigma_{+}\right) \backslash \Sigma_{+}$. For an integer $n \geq 1$ put

$$
N_{n} \stackrel{\text { def }}{=}\left\{j \in\{0, \ldots, n-1\}: f^{j}\left(x_{n}\right) \in B\left(\Sigma_{0}, \delta\right)\right\}
$$

and

$$
M_{n} \stackrel{\text { def }}{=}\left\{j \in\{0, \ldots, n-1\}: f^{j}\left(x_{n}\right) \in V_{+}\right\} .
$$

We have $\lim _{n \rightarrow+\infty}\left(M_{n}+N_{n}\right) / n=1$. Fix $n$ and let $k \geq 1$ and let $j_{1}, \ldots, j_{k}$ be all the integers $j \in\{1, \ldots, n-1\}$ such that $f^{j-1}\left(x_{n}\right) \notin V_{+}$and $f^{j}\left(x_{n}\right) \in V_{+}$. Similarly, let $k^{\prime}$ be the number of blocks of the trajectory of $x$ contained in $B\left(\Sigma_{0}, \delta\right)$. For each $i \in\{1, \ldots, k\}$ let $j_{i}^{\prime}$ be the largest integer $j \in\left\{j_{i}, \ldots, n-1\right\}$ such that for each $s \in\left\{j_{i}, \ldots, j\right\}$ we have $f^{s}\left(x_{n}\right) \in B(\Sigma, \delta)$. Then

$$
\sum_{i=1}^{k}\left(j_{i}^{\prime}-j_{i}+1\right)=M_{n} \quad \text { and } \quad \max \left\{k, k^{\prime}\right\} \leq n-\left(M_{n}+N_{n}\right) .
$$

Furthermore, for each $i \in\{1, \ldots, k\}$ so that $f^{j_{i}}\left(x_{n}\right) \in V_{+}$the point $f^{j_{i}-1}\left(x_{n}\right)$ is close to $f^{-1}(\Sigma)$ and we thus have

$$
\log \left|\left(f^{j_{i}^{\prime}-j_{i}+1}\right)^{\prime}\left(f^{j_{i}-1}\left(x_{n}\right)\right)\right| \leq\left(j_{i}^{\prime}-j_{i}+2\right) \chi_{\text {ess }}^{+}+C .
$$

Hence, for $C^{\prime}=2 C+\log \sup _{J}\left|f^{\prime}\right|$ we have

$$
\begin{aligned}
\log \left|\left(f^{n}\right)^{\prime}\left(x_{n}\right)\right| & \leq\left(M_{n}+k\right) \chi_{\text {ess }}^{+}+k C+N_{n} \varepsilon+k^{\prime} C+\left(n-M_{n}-N_{n}-k\right) \log \sup _{J}\left|f^{\prime}\right| \\
& \leq n \max \left\{\chi_{\text {ess }}^{+}, \varepsilon\right\}+\left(n-M_{n}-N_{n}\right) C^{\prime} .
\end{aligned}
$$

Since $\varepsilon>0$ is arbitrary, this implies

$$
\chi^{+}(J \backslash V)=\limsup _{n \rightarrow+\infty} \frac{1}{n} \log \left|\left(f^{n}\right)^{\prime}\left(x_{n}\right)\right| \leq \chi_{\text {ess }}^{+}
$$

finishing the proof of the lemma.

Lemma 11. Suppose $f$ is exceptional. Then for each $c \in f^{-1}\left(\Sigma_{+}\right) \backslash \Sigma_{+}$and $\varepsilon>0$ there is a periodic point $q$ close to $c$ such that

In particular, $\widetilde{\alpha}^{+} \geq \chi_{\text {ess }}^{+}$.

$$
\chi(q) \geq \chi_{\mathrm{ess}}(c)-\varepsilon
$$

Proof. Let $k \geq 1$ be the least integer such that $p=f^{k}(c) \in \Sigma$ is periodic, put $d \stackrel{\text { def }}{=} \operatorname{deg}_{f^{k}}(c)$ and let $\ell \geq 1$ be the period of $p$. Let $\delta>0$ be sufficiently small so that there is a local inverse $\phi$ of $f^{\ell}$ fixing $z=p$ defined on $B(p, \delta)$, in such a way that $\phi(B(p, \delta))$ is compactly contained in $B(p, \delta)$ and for some constant $\gamma_{0}>0$ and every $n \geq 1$ and $x \in \phi^{n}(B(p, \delta))$ we have $\left|\left(f^{n \ell}\right)^{\prime}(x)\right| \geq \gamma_{0} e^{n \ell \chi(p)}$. Since $c \notin$ 
$\Sigma$ there is a point $x \in B(p, \delta)$ and an integer $m \geq 1$ such that $f^{m}(x)=c$ and such that $\left(f^{m}\right)^{\prime}(x) \neq 0$. Let $\rho>0$ be sufficiently small so that the connected component $W$ of $f^{-m}(B(c, \rho))$ containing $x$ is such that $\bar{W} \subset B(p, \delta) \backslash\{p\}$ and $\gamma_{1}=$ $\inf _{z \in W}\left|\left(f^{m}\right)^{\prime}(z)\right|>0$. Then for every sufficiently large integer $n \geq 1$ there is a connected component $W_{n}$ of $f^{-k}\left(\phi^{n}(W)\right)$ compactly contained in $B(c, \rho)$. It follows that $W_{n}$ contains a periodic point $q_{n}$ of $f$ of period $k+n \ell+m$. We will now estimate its Lyapunov exponent. Since $\operatorname{dist}\left(\phi^{n}(W), p\right) \sim e^{-n \ell \chi(p)}$ we have $\operatorname{dist}\left(W_{n}, c\right) \sim e^{-n \ell \chi(p)}$, so there is a constant $\gamma_{2}>0$ such that for every $z \in W_{n}$ we have

$$
\left|\left(f^{k}\right)^{\prime}(z)\right| \geq \gamma_{2} e^{-n \ell \chi(p)(d-1) / d} .
$$

Therefore

$$
\left|\left(f^{k+n \ell+m}\right)^{\prime}\left(q_{n}\right)\right| \geq \gamma_{0} \gamma_{1} \gamma_{2} e^{-n \ell \chi(p) / d}=\gamma_{0} \gamma_{1} \gamma_{2} e^{-n \ell \chi_{\mathrm{ess}}(c)},
$$

and $\liminf _{n \rightarrow \infty} \chi\left(q_{n}\right) \geq \chi_{\text {ess }}(c)$.

Proof of Proposition 10. In view of Lemma 10 and Corollary 1, the proposition is a direct consequence of the Lemma 11.

We finally state the following immediate corollary.

Corollary 2. Let

$$
D \stackrel{\text { def }}{=} \max \operatorname{deg}_{f k(c)}(c),
$$

where the maximum is taken over all critical points $c \in f^{-1}(\Sigma) \backslash \Sigma$ and where $k(c)$ denotes the minimal integer such that $f^{k(c)}(c)$ is a periodic point. We have

$$
\alpha^{+} \leq D \widetilde{\alpha}^{+} .
$$

Proof of Theorem 1. The fact for every $\alpha \in\left[\alpha^{-}, \widetilde{\alpha}^{+}\right] \backslash\{0\}$ and every $\beta \in\left[\alpha, \widetilde{\alpha}^{+}\right]$ we have (6) and (7) is a direct consequence of Propositions 7 and 9. When $\alpha^{-}>0$, Proposition 9 implies that

$$
\operatorname{dim}_{\mathrm{H}}\left\{x \in J: \underline{\chi}(x)>0, \bar{\chi}(x)<\alpha^{-}\right\}=0 .
$$

On the other hand, in this case we also have $\widetilde{F}(0)=-\infty$, so $\operatorname{dim}_{\mathrm{H}} \mathcal{L}(0) \geq F(0)$ is trivially satisfied. When $\alpha^{-}=0$, this last inequality is given by Proposition 7 with $\alpha=\beta=0$.

The fact that for every $x$ in $J$ for which $\chi(x)$ exists we have either $\chi(x)=-\infty$ or $\chi(x) \geq \alpha^{-}$is given by [7, Lemma 9], and the fact that for every $x$ in $J \backslash \Sigma$ we have $\bar{\chi}(x) \leq \widetilde{\alpha}^{+}$is given by Proposition 10 . This completes the proof of the theorem.

\section{Appendix A. An alternative proof of the completeness of the spectrum. Specification property}

The purpose of this section is to give an alternative proof of Proposition 10. We will obtain this proposition as an easy consequence of Lemma 12 below. We shall conclude the Appendix with a more precise version of this lemma, corresponding to Bowen's periodic specification property, [4].

Lemma 12. Given a neighborhood $V \supset \Sigma$, for every $\varepsilon>0$ there exists a periodic point $p \in J \backslash \Sigma$, so that $\chi(p) \geq \chi^{+}(J \backslash V)-\varepsilon$. 
Proof. We first collect some preliminary definitions and results.

Recall that $n \geq 1$ is said to be a Pliss hyperbolic time for $x$ with exponent $\chi$ if

$$
\log \left|\left(f^{n-m}\right)^{\prime}\left(f^{m}(x)\right)\right| \geq(n-m) \chi \quad \text { for every } m=0, \ldots, n-1 .
$$

Given $\varepsilon>0$, by the telescope lemma, see for example [7, Lemma 9], there exist positive constants $K_{1}, R_{1}$ so that for every $r \in\left(0, R_{1}\right)$, every Pliss hyperbolic time $n$ for a point $x$ with exponent $\chi>0$, and every $m=0, \ldots, n-1$ we have

$$
\operatorname{diam} B_{m} \leq r K_{1} e^{-(n-m)(\chi-\varepsilon)},
$$

where

$$
B_{m} \stackrel{\text { def }}{=} f_{f^{m}(x)}^{-(n-m)}\left(B\left(f^{n}(x), r\right)\right) .
$$

Now let $r \in\left(0, \min \left\{R_{1}, \operatorname{dist}(\Sigma, \partial V\}\right)\right.$.

Let us briefly write $\chi^{+}=\chi^{+}(J \backslash V)$. By definition of $\chi^{+}$there exists $\widetilde{N} \geq 1$ so that for every $n \geq \widetilde{N}$ we have

$$
\sup _{x \in J \backslash V} \frac{a(x, n)}{n} \leq \chi^{+}+\varepsilon, \quad \text { where } a(x, n) \stackrel{\text { def }}{=} \log \left|\left(f^{n}\right)^{\prime}(x)\right| .
$$

On the other hand, for every large enough integer $n$ we can choose a point $x=x(n) \in J \backslash V$ so that $a(x, n)>n\left(\chi^{+}-\varepsilon / 2\right)$. Notice that we can assume that $n$ is a Pliss hyperbolic time for $x(n)$ with exponent $\chi^{+}-\varepsilon$ and satisfies $n \geq \tilde{N}$.

More precisely for the original $n$ let $n^{\prime} \in\{1, \ldots, n\}$ be an integer such that at $m=n^{\prime}$ the expression

$$
A(m) \stackrel{\text { def }}{=} a(x, m)-m\left(\chi^{+}-\varepsilon\right)
$$

attains its maximum. Clearly $n^{\prime}$ is a Pliss hyperbolic time for $x$. Moreover, since $A(n) \geq n \varepsilon / 2, A(0)=0$, and the function $\log \left|f^{\prime}\right|$ is upper bounded, we obtain $n^{\prime} \rightarrow \infty$ as $n \rightarrow \infty$. So we can replace $n$ by $n^{\prime}$, thus assuming we have a sequence of pairs $\left(\left(x_{j}, n_{j}\right)\right)_{j}$ so that $x_{j} \in J \backslash V, n_{j}$ is a Pliss hyperbolic time for $x_{j}$ with exponent $\chi^{+}-\varepsilon, n_{j} \geq \tilde{N}$, and $n_{j} \rightarrow \infty$ as $j \rightarrow \infty$. In the sequel we shall omit the index $j$.

We can assume that, possibly after slightly increasing $\varepsilon$, additionally we have $f^{n}(x) \notin V$. Indeed, let $m_{-} \in\{0, \ldots, n-1\}$ be the largest integer such that $y=$ $f^{m_{-}}(x) \notin V$. Since we assume that $n$ is a Pliss hyperbolic time for $x$ and since $y$ is close to a critical point, $\left|f^{\prime}(y)\right|$ is small and hence the number $n-m_{-}$must be large by (24). In particular, $n-m_{-} \geq$card $\Sigma$. Recall that $\Sigma$ contains periodic points together with their non-critical preimages. Let the forward trajectory of $y$ follow a periodic trajectory of a point $q \in \Sigma$. Denote by $N_{q}$ the least period of $q$. Then, by (24) we have

$$
a\left(f^{n-N_{q}}(x), N_{q}\right) \geq N_{q}\left(\chi^{+}-\varepsilon\right),
$$

which yields $\chi(q) \geq \chi^{+}-2 \varepsilon$ provided $V$ is small enough, where the factor 2 takes in account the distortion in a neighborhood of the trajectory of $q$. So in the case that $n$ is a Pliss hyperbolic time and $f^{n}(x) \in V$ we can consider the smallest integer $m_{+}>n$ for which $f^{m_{+}}(x) \notin V$. Then there exists an integer $m_{+}^{\prime}$ between $m_{+}$and $m_{+}+N_{q}$ which is a Pliss hyperbolic time for $x$ with exponent $\chi^{+}-3 \varepsilon$. Note that $m_{+}$and $m_{+}^{\prime}$ exist, provided $\chi^{+}-3 \varepsilon>0$ and $V$ is small enough.

By (27) for any point $y=f^{m}(x)$ with $m<n-\tilde{N}$ and $f(y) \notin V$ we have

$$
a(f(y), n-m-1) \leq(n-m-1)\left(\chi^{+}+\varepsilon\right) .
$$


Thus, together with (24) with $\chi=\chi^{+}-\varepsilon$, for any such $y$ we conclude

$$
\begin{aligned}
(n-m)\left(\chi^{+}-\varepsilon\right) & \leq a(y, n-m)=\log \left|f^{\prime}(y)\right|+a(f(y), n-m-1) \\
& \leq \log \left|f^{\prime}(y)\right|+(n-m-1)\left(\chi^{+}+\varepsilon\right)
\end{aligned}
$$

and hence

$$
\log \left|f^{\prime}(y)\right| \geq(n-m)\left(\chi^{+}-\varepsilon\right)-(n-m-1)\left(\chi^{+}+\varepsilon\right)>-(n-m) 2 \varepsilon .
$$

Therefore, such $y$ must be in some distance to critical points and satisfy

$$
\operatorname{dist}(y, \text { Crit }) \geq C \cdot e^{-(n-m) 2 \varepsilon},
$$

where $C$ is some positive constant.

As concluded before, $B \stackrel{\text { def }}{=} B\left(f^{n}(x), r\right)$ and $\Sigma$ are disjoint. Now we pull back $B$ and show that for $r$ small enough no pullback $B_{m}$ defined in (26) contains a critical point. To show this let us assume that the initially chosen $r$ also satisfies $r<C / K_{1} e^{\chi^{+}-4 \varepsilon}$ and that $\chi^{+}-4 \varepsilon>0$.

First, if $m$ satisfies $0 \leq m<n-\widetilde{N}$ we consider two cases:

1) $f^{m+1}(x) \notin V$ : Then (28) and (25) for $\chi=\chi^{+}-\varepsilon$ imply $B_{m} \cap$ Crit $=\varnothing$.

2) $f^{m+1}(x) \in V$ : Then (25) implies that $B_{m}$ is very small. So, if there were a critical point $c \in B_{m}$ then we would have $f(c) \in \Sigma$. Since $\Sigma$ is forward invariant, this would imply $\Sigma \cap B\left(f^{n}(x), r\right) \neq \varnothing$ which is a contradiction. Hence $B_{m} \cap$ Crit $=\varnothing$.

Second, in the remaining finite number of cases if $m$ satisfies $n-\widetilde{N} \leq m<n$ we can assure, possibly after decreasing $r$, not depending of $(x, n)$ (possible since $n$ are Pliss hyperbolic times with common $\chi$ ), that $B_{m} \cap$ Crit $=\varnothing$. Thus, we can conclude that none of the pullbacks $B_{m}$ captures a critical point and therefore $f^{n-m}$ is univalent on $B_{m}$ for every $m=0, \ldots, n-1$.

Given $r$, by Lemma 3 there exists $\delta>0$ and positive integers $N, M$, $i$, and $j$ with $N \leq M$ and $0 \leq i, j \leq M$, and a point $z \in f^{-j}\left(f^{i}(x)\right)$ such that $A \stackrel{\text { def }}{=} f^{-N}(z)$ is $r / 2$-dense in $J$ and satisfies $\operatorname{dist}\left(f^{k}(A)\right.$, Crit $) \geq \delta$ for every $k=0, \ldots, N+j-1$.

Now we can choose $\delta^{\prime} \in(0, \delta)$ independent of $x$ so that for this point we have $z \in f^{-j}\left(f^{i}(x)\right)$ and every $k=0, \ldots, N+j-1$ we have

$$
f^{k}\left(f^{-N}\left(\operatorname{Comp}_{z} f^{-j}\left(B\left(f^{i}(x), \delta^{\prime}\right)\right)\right)\right) \cap \text { Crit }=\varnothing,
$$

yielding that $f^{N+j}$ is univalent on $B(w)$ defined by

$$
B(w) \stackrel{\text { def }}{=} f_{w}^{-(N+j)}\left(B\left(f^{i}(x), \delta^{\prime}\right)\right)
$$

for every $w \in A$ as well as

$$
\operatorname{diam} B(w) \leq r / 3
$$

Thus, if $n$ is large enough so that $r K_{1} e^{-(n-i)\left(\chi^{+}-2 \varepsilon\right)}<\delta^{\prime}$ then with $y \stackrel{\text { def }}{=} f^{i}(x)$ and using (25) with $\chi=\chi^{+}-\varepsilon$ we obtain

$$
f_{y}^{-(n-i)}\left(B\left(f^{n}(x), r\right)\right) \subset B\left(y, \delta^{\prime}\right) .
$$

and hence for some choice of $w \in A$ (that will in general depend on $f^{n}(x)$ ) we have

$$
\widetilde{B}(w) \stackrel{\text { def }}{=} f^{-(n-i+N+j)}\left(B\left(f^{n}(x), r\right)\right) \subset B\left(f^{n}(x), 5 r / 6\right)
$$


and $f^{n-i+N+j}$ is univalent on $\widetilde{B}(w)$. Hence the latter set contains a periodic point $p$. Using (24) and distortion estimates, it can be achieved that $\chi(p) \geq \chi^{+}-3 \varepsilon$, if $n$ is large enough. This proves the lemma.

Remark. The idea of the proof of Lemma 12 is taken from an unpublished note [13], where the supremum in (23) was taken over all $x \in J$ but allowing the periodic point $p$ to belong to $\Sigma$. The proof was simpler in that case as it did not rely on the telescope result (25). The result in [13] has been applied and referred to in [8].

Another proof of the weaker statement, that is, allowing $p \in \Sigma$, can be given by constructing a measure $\mu$ that is an accumulation of the sequence of measures $\frac{1}{n} \sum_{k=0}^{n-1} \delta_{f^{k}(x)}$ as $n \rightarrow \infty$, where $\delta_{y}$ denotes the Dirac measure supported at $y$. Here $x$ and $n$ should be chosen to give an approximation of $\chi^{+}$by $a(x, n) / n$. One finds $p$ using Katok's method, see [16, Chapter 11.6]. This easy 'ergodic' proof is in fact a part of the proof in Section 5.3.

Notice that the proof in Section 5.3 does not yield the periodic specification property below because it bases on a specification of, maybe short, special sub-blocks of a given piece of a trajectory.

Proof of Proposition 10. By Lemma 12 for every $x \notin \Sigma$ we have

$$
\bar{\chi}(x) \leq \sup \{\chi(p): p \in J \backslash \Sigma \text { periodic repelling }\} .
$$

By Corollary 1 this bound is less than or equal to $\widetilde{\alpha}^{+}$. This proves the proposition.

Proposition 11. For every $V$ and $\varepsilon$ as in Lemma 12 there exist an integer $N>0$ and $\varepsilon_{1}>0$ such that for every point $x \in J \backslash V$ and $n \geq N$ with $f^{n}(x) \notin V$ satisfying $a(x, n) \geq \chi^{+}(J \backslash V)-\varepsilon_{1}$, there exists an integer $m \in\{n(1-\varepsilon), \ldots, n\}$ and a periodic point $p \in J$ of period at most $n+N$, such that:

1. $\chi(p) \geq \chi^{+}(J \backslash V)-\varepsilon$;

2. $\operatorname{dist}\left(f^{j}(x), f^{j}(p)\right) \leq e^{-(m-j)\left(\chi^{+}(J \backslash V)-\varepsilon\right)}$ for all $j=4,5, \ldots, m$.

Proof. We just look more carefully at the proof of Lemma 12. Consider an arbitrary small $t>0$. Assume that $x$ and $n$ satisfy $a(x, n) \geq n\left(\chi^{+}-t \varepsilon\right)$.

By definition of $\chi^{+}=\chi^{+}(J \backslash V)$ we can also assume, compare $(27)$, that $a(x, m) \leq$ $m\left(\chi^{+}+t \varepsilon\right)$ for all $m \in\{N(t \varepsilon), \ldots, n-1\}$, for some constant $N(t \varepsilon)$ depending only on $t \varepsilon$.

Then we find a number $n^{\prime} \leq n$ that is a Pliss hyperbolic time for $x$ with exponent $\chi^{+}-\varepsilon$, as in the proof of Lemma 12. To estimate $n^{\prime}$, notice that

$$
A(n)=a(x, n)-n\left(\chi^{+}-\varepsilon\right) \geq n \varepsilon(1-t),
$$

whereas

$$
A(m)=a(x, m)-m\left(\chi^{+}-\varepsilon\right) \leq m\left(\chi^{+}+t \varepsilon\right)-m\left(\chi^{+}-\varepsilon\right)=m \varepsilon(1+t) .
$$

Hence, for $m<n \frac{1-t}{1+t}$, we obtain $A(m)<A(n)$. Notice also that for $m \leq N(t \varepsilon)$ we have $A(m)<n \varepsilon(1-t) \leq A(n)$ for $n$ large enough since sup $\left|f^{\prime}\right|<\infty$. In consequence, the positive integer $n^{\prime}=m$ maximizing $A(m)$ is bigger or equal to $n \frac{1-t}{1+t}$. Finally we choose $t$ so that $\frac{1-t}{1+t}>1-\varepsilon$.

Next in the proof of Lemma 12 we have increased $n^{\prime}$ to achieve $f^{n^{\prime}}(x) \notin V$. Here the increase is not beyond $n$ since we have already assumed $f^{n}(x) \notin V$. 
The rest of the proof is the same. The mysterious indices $j=4,5, \ldots$ in the assertion comes from Lemma 1 implying that at most fourth iteration of any point has a backward branch omitting critical points, hence $i \leq 4$.

Acknowledgement. KG has been supported by the Alexander von Humboldt Foundation Germany, CNPq and FAPERJ, Brazil. The research of FP and MR were supported by the EU FP6 Marie Curie programmes SPADE2 and CODY and by the Polish MNiSW Grant NN201 022233 'Chaos, fraktale i dynamika konforemna'. JR-L was partially supported by FONDECYT grant N 1100922

\section{References}

[1] Barreira, L., Ya. Pesin, and J. Schmeling: On a general concept of multifractality. Multifractal spectra for dimensions, entropies, and Lyapunov exponents. Multifractal rigidity. Chaos 7, 1997, 27-38.

[2] Barreira, L., B. Saussol, and J. Schmeling: Distribution of frequencies of digits via multifractal analysis. - J. Number Theory 97, 2002, 410-438.

[3] Besicovitch, A.: On the sum of digits of real numbers represented in the dyadic system. Math. Ann. 110, 1934, 321-330.

[4] Bowen, R.: Periodic points and measures for Axiom A diffeomorphisms. - Trans. Amer. Math. Soc. 154, 1971, 377-397

[5] Denker, M., and M. Urbanski: On the existence of conformal measures. - Trans. Amer. Math. Soc. 328, 1991, 563-587.

[6] Doundy, A., and J.H. HubBard: A proof of Thurston's topological characterization of rational functions. - Acta Math. 171, 1993, 263-297.

[7] Gelfert, K., F. Przytycki, and M. Rams: Lyapunov spectrum for rational maps. - Math. Ann. 348, 2010, 965-1004.

[8] Gooransarab, H.: An upper estimate for characteristic exponent of polynomials. - PhD dissertation, Purdue University, 1998.

[9] Makarov, N., and S. Smirnov: Phase transition in subhyperbolic Julia sets. - Ergodic Theory Dynam. Systems 16, 1996, 125-157.

[10] Makarov, N., and S. Smirnov: On the "thermodynamics" of rational maps I. Negative spectrum. - Comm. Math. Phys. 211, 2000, 705-743.

[11] PÉrez-Marco, R.: Fixed points and circle maps. - Acta Math. 179, 1997, 243-294.

[12] Pesin, YA.: Dimension theory in dynamical systems: Contemporary views and applications. - Chicago Lectures in Math., Chicago Univ. Press, 1997.

[13] Przytycki, F.: A letter to A. Eremenko. - http://www.impan.pl/ feliksp/experem.pdf, 1994.

[14] PRzytycki, F.: Conical limit sets and Poincaré exponent for iterations of rational functions. - Trans. Amer. Math. Soc. 351, 1999, 2081-2099.

[15] Przytycki, F., J. Rivera-Letelier, and S. Smirnov: Equality of pressures for rational functions. - Ergodic Theory Dynam. Systems 24, 2004, 891-914.

[16] Przytycki, F., and M. URBAnski: Conformal fractals: Ergodic theory methods. - London Math. Soc. Lecture Note Ser. 371, Cambridge Univ. Press, 2010.

[17] Walters, P.: An introduction to ergodic theory. - Grad. Texts in Math. 79, Springer, 1981.

[18] Weiss, H.: The Lyapunov spectrum for conformal expanding maps and Axiom-A surface diffeomorphisms. - J. Statist. Phys. 95, 1999, 615-632. 